\title{
Numerical simulation of group combustion of pulverized coal
}

\author{
A. Bermúdez ${ }^{\mathrm{a}, *}$, J.L. Ferrín ${ }^{\mathrm{a}}$, A. Liñán ${ }^{\mathrm{b}}$, L. Saavedra ${ }^{\mathrm{a}}$ \\ a Departamento de Matemática Aplicada, Universidad de Santiago de Compostela, 15782 Santiago de Compostela, Spain \\ ${ }^{\mathrm{b}}$ E.T.S. Ingenieros Aeronáuticos, Pl. Cardenal Cisneros 3, Universidad Politécnica de Madrid, 28040 Madrid, Spain
}

\begin{abstract}
A B S T R A C T
A mathematical model for the group combustion of pulverized coal particles was developed in a previous work. It includes the Lagrangian description of the dehumidification, devolatilization and char gasification reactions of the coal particles in the homogenized gaseous environment resulting from the three fuels, $\mathrm{CO}, \mathrm{H}_{2}$ and volatiles, supplied by the gasification of the particles and their simultaneous group combustion by the gas phase oxidation reactions, which are considered to be very fast. This model is complemented here with an analysis of the particle dynamics, determined principally by the effects of aerodynamic drag and gravity, and its dispersion based on a stochastic model. It is also extended to include two other simpler models for the gasification of the particles: the first one for particles small enough to extinguish the surrounding diffusion flames, and a second one for particles with small ash content when the porous shell of ashes remaining after gasification of the char, non structurally stable, is disrupted.

As an example of the applicability of the models, they are used in the numerical simulation of an experiment of a non-swirling pulverized coal jet with a nearly stagnant air at ambient temperature, with an initial region of interaction with a small annular methane flame. Computational algorithms for solving the different stages undergone by a coal particle during its combustion are proposed. For the partial differential equations modeling the gas phase, a second order finite element method combined with a semiLagrangian characteristics method are used. The results obtained with the three versions of the model are compared among them and show how the first of the simpler models fits better the experimental results.
\end{abstract}

\section{Introduction}

In 2008 , fossil fuels accounted for $86 \%$ of all energy produced in the world (see [1]) and will continue to be so for the foreseeable future. Among all the fossil fuels, coal is the main source of energy used to generate electricity. Pulverized coal fired furnaces are extensively used in power generation worldwide. Because of economic reasons and more restrictive legislations in terms of pollutant emissions, major efforts are being invested in making the use of pulverized coal more efficient and environmentally acceptable. In particular, they have been focused on the development of multidimensional mathematical models that can be used to design and optimize the combustion processes. With this purpose, in recent years several CFD codes have been developed by research organizations [2-7] and by specialized commercial companies. An extensive overview of the modeling technology as applied to fossil-fuel combustion processes was made by Eaton et al. [8]. It includes a review of the state-of-the-art of the various components or submodels required in a combustion model, the consideration of kinds of data required to evaluate and validate the predictions of combustion codes, and a summary of representative applications. As mentioned by Korytnyi et al. [9] or Williams et al. [10], the main limitation in CFD modeling of coal combustion is the use of simplified models for devolatilization, gasification of char and burning of volatile matters. This limitation comes from the need of a good characterization of the parameters involved in the different submodels.

In a previous work by Bermúdez et al. [11], a model to be referred in the following as the BFL model (as the acronym of Bermúdez, Ferrín and Liñán) for the description of the combustion of coal particles in pulverized coal combustion furnaces was developed. The resulting mathematical model, summarized and generalized below, is based on the realistic assumption that the gas phase oxidation reactions of the volatiles, $\mathrm{H}_{2}$ and $\mathrm{CO}$, supplied to the gas phase by the gasification of the coal particles, are very fast. The model was aimed to deal with the combustion of coal particles with a wide range of volatiles, humidity and ash content, as well as sizes.

A Lagrangian description was proposed to follow the individual time evolution of the coal particles, represented by an appropriate 


\section{Nomenclature}

a initial radius of the particle

$B_{i} \quad$ frequency factor of reaction $i$

$c_{p} \quad$ specific heat at constant pressure of the gas mixture

$c_{s} \quad$ specific heat of the coal particle

$\mathcal{D}$ diffusion coefficient of the gas phase

$\mathcal{D}_{e} \quad$ effective diffusion coefficient through the porous parti-

cle

$E_{i} \quad$ activation energy of reaction $i$

$f^{e} \quad$ gas phase homogenized source of energy, per unit volume and time

$f_{A}^{\mathrm{m}} \quad$ gas phase homogenized source of mass of species $A$, per unit volume and time

$h_{T} \quad$ thermal enthalpy

$k$ thermal conductivity

$q_{i} \quad$ heat released per unit of mass gasified by the ith reaction heat flux reaching the particle surface by conduction radiative heat flux reaching the particle universal gas constant radius of the shrinking core cross-over temperature of the particle temperature of the particle mass fraction of $\alpha$ in the ambient gas radius of the particle changing with time nondimensional gasification rate of the particle, scaled with $4 \pi \rho_{g} a \mathcal{D}$

nondimensional contribution to $\lambda$ of the reaction $i$ region of the flow field without oxygen region of the flow field with oxygen diffusion flames enclosing $\Omega_{F}$ density of the gas mixture density of the coal particle sample, in the variable environment created collectively by them in the gas phase. This environment results from the sources of reactants, products and heat due to the volatilization and gasification reactions taking place within the particles. The volatiles emitted by the particles, and the $\mathrm{CO}$ and $\mathrm{H}_{2}$ resulting from gasification will react with the $\mathrm{O}_{2}$ encountered in the gas phase to produce $\mathrm{H}_{2} \mathrm{O}$ and $\mathrm{CO}_{2}$. These species may diffuse back to the particles to participate, together with $\mathrm{O}_{2}$, in the gasification of the char, mainly after the dehumidification and devolatilization stages occurring in the chamber early in the coal particle history.

If we want complete combustion of the coal, the design conditions of the furnace should ensure that the characteristic times of the gas phase oxidation reactions, at the high flame temperatures, are small compared with the typical residence time in the furnace. In this case, we can obtain a good description of the temperature and concentration fields of the main species using the assumption that the gas phase oxidation reactions of the volatiles, $\mathrm{H}_{2}$ and $\mathrm{CO}$ are irreversible and infinitely fast. Then, they must occur simultaneously in the same flame sheet, $\Gamma_{F}$ (see Fig. 1), corrugated and fluctuating due to the turbulent flow, which encloses a region $\Omega_{F}$ without oxygen where the particles are sources for the water vapor and volatiles, as well as for the $\mathrm{CO}$ and $\mathrm{H}_{2}$ produced by the char gasification by $\mathrm{CO}_{2}$ and $\mathrm{H}_{2} \mathrm{O}$. The gas phase reactions take place in a thin diffusion flame $\Gamma_{F}$, in the form of group combustion of the reactants generated in $\Omega_{F}$. The position of $\Gamma_{F}$ depends on the competition for the $\mathrm{O}_{2}$ of these three fuels, which are produced in different proportions due to the particle history. These fuels differ in the stoichiometry and heat release of their oxidation reactions; these also result in different ratios of their reaction products $\mathrm{H}_{2} \mathrm{O}$ and $\mathrm{CO}_{2}$, which are involved in the char gasification reactions. The differences in the oxidation reactions of the three fuels, and in the spatial location of their generation, complicate significantly the analysis of pulverized coal combustion, even in the realistic limit of fast oxidation reactions.

The oxidation reactions could also take place, as considered in [11], in individual flame sheets surrounding, or inside, the particles in the outer region $\Omega_{O}$ where the oxygen in the gas environment of the particles has not been depleted as in $\Omega_{F}$. For this to be the case the particle size has to be large compared with the thickness of a typical CO/air flame; otherwise the gasification reaction will not occur in closed diffusion flames around the particles but will only take place in a distributed form in the collective gas environment, at a rate fast enough to ensure that the reactant concentrations of the volatiles, $\mathrm{H}_{2}$ and $\mathrm{CO}$ are small compared with those of the products $\mathrm{CO}_{2}$ and $\mathrm{H}_{2} \mathrm{O}$.

For the dehumidification and devolatization reactions of the particles we have used reaction rates of the Arrhenius type, but the analysis can be easily generalized for other more complex models. The included char gasification model consists of three overall reactions corresponding to the char gasification by $\mathrm{O}_{2}$, $\mathrm{CO}_{2}$ and $\mathrm{H}_{2} \mathrm{O}$ that must diffuse into the interior of the particles from the gas environment. The overall rate will typically involve

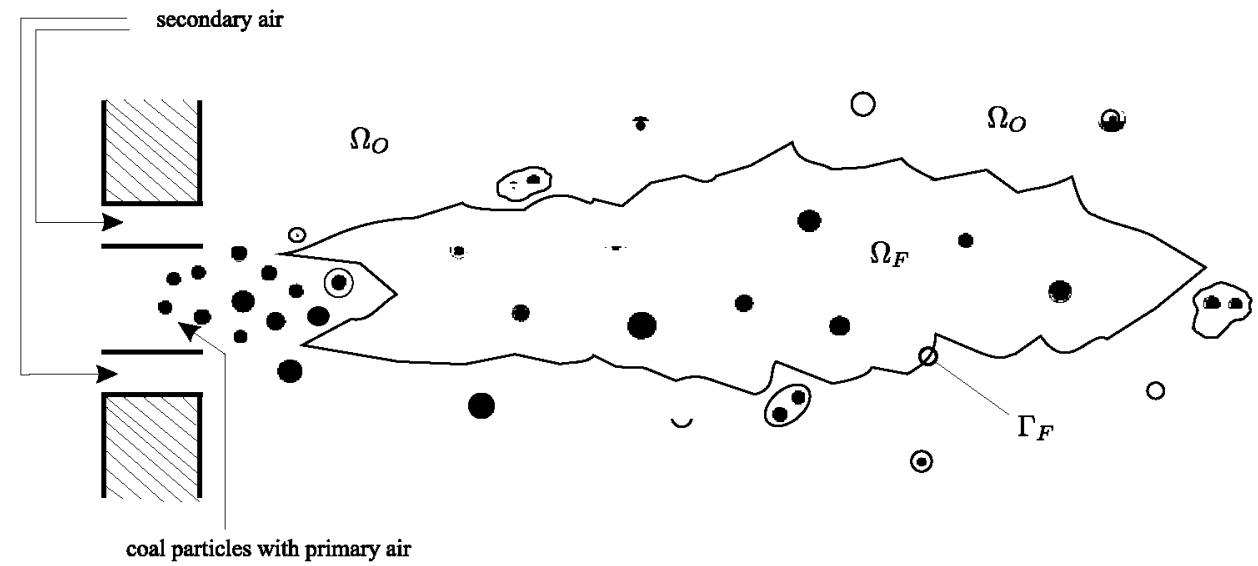

Fig. 1. Diffusion flame. 
a large activation temperature for the overall reaction time; in this case, there is a cross-over temperature of the particle, $T_{c}$ (that we consider to differ little for the three reactions), below which the gasification time is large compared with the diffusion time and the gasification reactions can be neglected, and above which the rates are so fast that the gasification reactions are not kinetically but diffusion-controlled.

We have shown in [11] that in this regime of diffusion-controlled gasification, for coal particles with a moderately large content of ashes, the gasification takes place in a shrinking core with radius $r_{c}(t)$ smaller than the original particle radius $a$. The $\mathrm{O}_{2}$, $\mathrm{CO}_{2}$ and $\mathrm{H}_{2} \mathrm{O}$ have to diffuse across the ash layer to reach the char gasification layer with a rate that determines the rate of decrease of the radius $r_{C}$ of the shrinking core. In this paper we complement the analysis in [11] to deal also with coal particles with small ash content, as it is typically considered in the coal combustion literature. In this case, the ash layer remaining after char gasification would have a large porosity and is continually disrupted because it is structurally unstable, so that the particle radius, $a$, is now variable with time and equal to $r_{c}$ and the overall particle gasification rate takes a simplified form that will be given below.

Under diffusion-controlled conditions and in a typically turbulent flow, the gas phase oxidation reactions taking place in strongly fluctuating thin layers have to be dealt with by using Shvab-Zeldovich coupling functions, which are combinations of the reacting species having no sources due to the gas phase chemical reactions. They are complemented with the asymptotic assumption that there is no oxygen in $\Omega_{F}$ and no volatiles, CO or $\mathrm{H}_{2}$ in $\Omega_{\mathrm{O}}$. To account for the competition of the three species for the $\mathrm{O}_{2}$ in the diffusion flame, the Burke-Schumann analysis has been generalized in a new original way.

The BFL model presented in [11] and generalized below provides the framework not existing before for the direct numerical simulation of laminar and transitional group combustion of swirling and non-swirling pulverized coal jets. In addition, it provides the framework to obtain the modeling equations required for the engineering simulation of the turbulent combustion (with fast gas phase reactions) in pulverized coal furnaces. This model still to be developed would be very useful in engineering design. Because the direct numerical simulation being too costly or impossible, the analysis uses $k-\epsilon$ schemes to describe the turbulent diffusivity of the reactants and either presumed PDF's to calculate the mean reaction rates in diffusion-controlled gas phase reactions or an eddy break-up model of not easy, to say the least, justification. The BFL model is extended here to account for the description of the particle dynamics, including particle diffusivity due to turbulence and also to account for the description of the gasification of coal particles with small ash content.

The goal of this paper is to show how the BFL model can be applied in the numerical simulation of group combustion. Here it is used for the analysis of a simple example of the group combustion of a non-swirling pulverized coal jet. This will be done for the general BFL model (where gas phase reaction rates are assumed to be fast to avoid the extinction of the diffusion flame in the vicinity of the particle) and for the two mentioned simpler cases. In the first one we shall deal with coal particles smaller than a critical diameter, lower than $100 \mu \mathrm{m}$, for the extinction of the diffusion flame. In this case, the gas phase reactions are not fast enough to take place in the vicinity of the particle. However, they could occur in the near wake, without affecting to its gasification rate, or mainly in the homogenized region of the gas mixture where the oxidation reactions behave as sufficiently fast to be near completion, with small values of the mass fractions of the species being oxidized, thus ensuring the validity of the Burke-Schumann hypothesis. The second one concerns the situation where the content of ashes in the particle is small and disruption of the very porous ash layer in the surface of the particle occurs. Thus the radius of the particle changes during the char gasification. This situation is very important in pulverized coal furnaces when high quality coals (as anthracite or sub-bituminous coals) are being burnt.

Thus, this work can be seen as a second part of the paper by Bermúdez et al. [11], because models for the above two simple cases are presented, as well as an overall numerical algorithm and computational results. Having this in mind, the experimental flame proposed by Hwang et al. [12,13] is solved with our models, and numerical results obtained are compared among them and with experimental data.

This paper is organized as follows. The physico-chemical model describing the combustion of a coal particle as well as a summary of the models for the gas and solid phases are presented in Section 2 . In that section we also show the expressions of the sources of mass and energy to the gas phase coming from the solid phase. The model for the coal particle motion is described in Section 3. Finally Section 4 shows the algorithms used to solve the models and numerical results concerning an experiment presented in Hwang et al. [12,13]. Even if the experiment conditions are not fully described in these papers and some uncertainties remain, the numerical results are in good agreement with the experimental measurements. This fact makes the introduced models very promising in their application to pulverized coal boilers which is the subject of current research by the authors. We finish with some conclusions.

\section{The combustion model}

The BFL combustion model considers a simplified kinetic model consisting of the following physico-chemical processes within the porous particles, leading to their gasification with production of volatiles, $\mathrm{CO}$ and $\mathrm{H}_{2}$

\begin{tabular}{ll}
\hline$\underline{1}$ & $\mathrm{CO}_{2}+\mathrm{C}_{(s)} \rightarrow 2 \mathrm{CO}+\left(q_{1}\right)$ \\
$\underline{2}$ & $\frac{1}{2} \mathrm{O}_{2}+\mathrm{C}_{(s)} \rightarrow \mathrm{CO}+\left(q_{2}\right)$ \\
$\underline{3}$ & $\mathrm{H}_{2} \mathrm{O}+\mathrm{C}_{(s)} \rightarrow \mathrm{CO}+\mathrm{H}_{2}+\left(q_{3}\right)$ \\
$\underline{4}$ & $\mathrm{~V}_{(s)} \rightarrow \mathrm{V}_{(g)}+\left(q_{4}\right)$ \\
$\underline{5}$ & $\mathrm{H}_{2} \mathrm{O}_{(s)} \rightarrow \mathrm{H}_{2} \mathrm{O}_{(g)}+\left(q_{5}\right)$ \\
\hline
\end{tabular}

where index $s$ refers to the solid phase and $g$ to the gas phase, and of the following gas phase oxidation reactions:

\begin{tabular}{ll}
\hline$\underline{6}$ & $\mathrm{CO}+\frac{1}{2} \mathrm{O}_{2} \rightarrow \mathrm{CO}_{2}+\left(q_{6}\right)$ \\
$\underline{7}$ & $\mathrm{~V}_{(g)}+v_{1} \mathrm{O}_{2} \rightarrow v_{2} \mathrm{CO}_{2}+v_{3} \mathrm{H}_{2} \mathrm{O}+v_{4} \mathrm{SO}_{2}+\left(q_{7}\right)$ \\
$\underline{8}$ & $\mathrm{H}_{2}+\frac{1}{2} \mathrm{O}_{2} \rightarrow \mathrm{H}_{2} \mathrm{O}+\left(q_{8}\right)$ \\
\hline
\end{tabular}

Reactions (1), (3) and (5) are endothermic whereas (2), (6), (7) and (8) are exothermic reactions. On the other hand, because of the lack of data, $q_{4}$ is considered to be zero.

All the volatiles are considered like one single molecule

$\mathrm{V}_{(g)}=C_{\kappa_{1}} H_{\kappa_{2}} O_{\kappa_{3}} S_{\kappa_{4}}$,

of molecular mass $M_{\mathrm{vol}}$, where coefficients $\kappa_{1}, \kappa_{2}, \kappa_{3}$ and $\kappa_{4}$ are deduced from the ultimate analysis of the coal. The stoichiometric coefficients $v_{i}$ in reaction (7) are calculated in terms of the volatiles composition using these expressions:

$$
\begin{array}{ll}
v_{1}=\left(2 \kappa_{1}+\kappa_{2} / 2+2 \kappa_{4}-\kappa_{3}\right) / 2, & v_{2}=\kappa_{1}, \\
v_{3}=\kappa_{2} / 2, & v_{4}=\kappa_{4} .
\end{array}
$$


The combustion models presented in this paper consist of two coupled models: the gas phase model and the solid or discrete phase model. On the one hand the gas phase model determines the atmosphere where particles are burnt (it is common to the three cases) and, on the other hand, the solid phase model provides the mass and energy sources to the gas.

\subsection{Gas phase model}

Let $\mathcal{L}_{g}$ be the differential operator defined by

$\mathcal{L}_{g}(u):=\frac{\partial\left(\rho_{g} u\right)}{\partial t}+\nabla \cdot\left(\rho_{g} u \mathbf{v}_{g}\right)-\nabla \cdot\left(\rho_{g} \mathcal{D} \nabla u\right)$,

where $\mathcal{D}$ is a gas phase diffusion coefficient which, for simplicity, will be considered to be the same for all species and equal to the thermal diffusivity. In order to facilitate the description of the effects of the fast gas phase oxidation reactions when they take place in thin reaction layers, we use a generalization of the Burke-Schumann procedure, accounting for the competition of the volatiles, $\mathrm{CO}$ and $\mathrm{H}_{2}$ for their oxidation with $\mathrm{O}_{2}$. From the eight conservation equations for the gaseous species and energy we can obtain the following five linear combinations of these equations to eliminate the terms associated to gas phase reactions. They include homogenized sources coming from the gasification of the particles.

$$
\begin{aligned}
& \mathcal{L}_{g}\left(\beta_{1}^{g}\right)=f_{\mathrm{O}_{2}}^{\mathrm{m}}-\frac{4}{7} f_{\mathrm{CO}}^{\mathrm{m}}-\frac{32 v_{1}}{M_{\mathrm{vol}}} f_{\mathrm{V}}^{\mathrm{m}}-8 f_{\mathrm{H}_{2}}^{\mathrm{m}}, \\
& \mathcal{L}_{\mathrm{g}}\left(\beta_{2}^{g}\right)=f_{\mathrm{CO}_{2}}^{\mathrm{m}}+\frac{11}{7} f_{\mathrm{CO}}^{\mathrm{m}}+\frac{44 v_{2}}{M_{\mathrm{vol}}} f_{\mathrm{V}}^{\mathrm{m}}, \\
& \mathcal{L}_{g}\left(\beta_{3}^{g}\right)=f_{\mathrm{H}_{2} \mathrm{O}}^{\mathrm{m}}+\frac{18 v_{3}}{M_{\mathrm{vol}}} f_{\mathrm{V}}^{\mathrm{m}}+9 f_{\mathrm{H}_{2}}^{\mathrm{m}}, \\
& \mathcal{L}_{g}\left(\beta_{4}^{g}\right)=f_{\mathrm{SO}_{2}}^{\mathrm{m}}+\frac{64 v_{4}}{M_{\mathrm{vol}}} f_{\mathrm{V}}^{\mathrm{m}}, \\
& \mathcal{L}_{g}\left(H^{g}\right)=f^{\mathrm{e}}+q_{6} f_{\mathrm{CO}}^{\mathrm{m}}+q_{7} f_{\mathrm{V}}^{\mathrm{m}}+q_{8} f_{\mathrm{H}_{2}}^{\mathrm{m}}-\nabla \cdot \mathbf{q}_{\mathrm{rg}} .
\end{aligned}
$$

These are conservation equations for the following scalars of the Shvab-Zeldovich type:

$$
\begin{aligned}
& \beta_{1}^{g}=Y_{\mathrm{O}_{2}}^{\mathrm{g}}-\frac{4}{7} Y_{\mathrm{CO}}^{\mathrm{g}}-\frac{32 v_{1}}{M_{\mathrm{vol}}} Y_{\mathrm{V}}^{\mathrm{g}}-8 Y_{\mathrm{H}_{2}}^{\mathrm{g}}, \\
& \beta_{2}^{\mathrm{g}}=Y_{\mathrm{CO}_{2}}^{\mathrm{g}}+\frac{11}{7} Y_{\mathrm{CO}}^{\mathrm{g}}+\frac{44 v_{2}}{M_{\mathrm{vol}}} Y_{\mathrm{V}}^{\mathrm{g}}, \\
& \beta_{3}^{g}=Y_{\mathrm{H}_{2} \mathrm{O}}^{\mathrm{g}}+\frac{18 v_{3}}{M_{\mathrm{vol}}} Y_{\mathrm{V}}^{\mathrm{g}}+9 Y_{\mathrm{H}_{2}}^{\mathrm{g}}, \\
& \beta_{4}^{\mathrm{g}}=Y_{\mathrm{SO}_{2}}^{\mathrm{g}}+\frac{64 v_{4}}{M_{\mathrm{vol}}} Y_{\mathrm{V}}^{\mathrm{g}}, \\
& H^{g}=h_{\mathrm{T}}^{\mathrm{g}}+q_{6} Y_{\mathrm{CO}}^{g}+q_{7} Y_{\mathrm{V}}^{g}+q_{8} Y_{\mathrm{H}_{2}}^{g} .
\end{aligned}
$$

In the Burke-Schumann limit of infinite gas phase reaction rates, (3)-(7) are complemented with the condition of non-coexistence of $\mathrm{O}_{2}$ and the species being oxidized. Then once we have solved (3), the diffusion flame surface, $\Gamma_{F}$, is given by $\beta_{1}^{g}=0$ and the value of $\beta_{1}^{g}$ will determine two regions of the gas domain and the way to recover the mass fractions and the enthalpy from the Shvab-Zeldovich variables:

1. If $\beta_{1}^{g}>0$ we are in domain $\Omega_{O}$. This implies that $Y_{\mathrm{V}}^{g}=Y_{\mathrm{H}_{2}}^{g}=Y_{\mathrm{Co}}=0$ and using (8)-(12) we obtain

$\mathrm{Y}_{\mathrm{O}_{2}}^{\mathrm{g}}=\beta_{1}^{\mathrm{g}}, \quad Y_{\mathrm{CO}_{2}}^{\mathrm{g}}=\beta_{2}^{\mathrm{g}}, \quad Y_{\mathrm{H}_{2} \mathrm{O}}^{\mathrm{g}}=\beta_{3}^{\mathrm{g}}$,

$\mathrm{Y}_{\mathrm{SO}_{2}}^{\mathrm{g}}=\beta_{4}^{\mathrm{g}}, \quad h_{T}^{\mathrm{g}}=H^{g}$.

2. If $\beta_{1}^{g}<0$ we are in domain $\Omega_{F}$ so $Y_{\mathrm{O}_{2}}^{g}=0$ and reactions (6)-(8) cannot occur. In this case to determine the mass fractions and the enthalpy we need to solve two additional equations but without gas phase reaction terms. For example, we can choose the equations

$\mathcal{L}_{\mathrm{g}}\left(\mathrm{Y}_{\mathrm{V}}^{\mathrm{g}}\right)=f_{\mathrm{V}}^{\mathrm{m}}$ in $\Omega_{\mathrm{F}}$,

$\mathcal{L}_{\mathrm{g}}\left(\mathrm{Y}_{\mathrm{H}_{2}}^{\mathrm{g}}\right)=f_{\mathrm{H}_{2}}^{\mathrm{m}}$ in $\Omega_{\mathrm{F}}$,

which must be integrated using boundary conditions $Y_{\mathrm{V}}^{g}=0$ and $Y_{\mathrm{H}_{2}}^{\mathrm{g}}=0$ on surface $\Gamma_{F}$.

\subsection{Particle gasification model}

The discrete phase model developed in [11] was based on a Lagrangian computation of the temperature and density of each coal particle throughout its trajectory, assuming the inequalities $L \gg l_{c} \gg l_{p} \gg a$ among the length scales, $L$ of the burner, $l_{c}$ of the computational cell, $I_{p}$ of the interparticle distance and $a$ the radius of the coal particle. Furthermore, we supposed that the particles were spherical and its radius and its ash density remained constant during combustion. Thus the model is valid for particles with high ash content.

As it was mentioned in the introduction, for the description of the generation of volatiles and evaporation of moisture we consider the same model as in the general case (see [11] for further details), namely, the evolution in time of $\rho_{\mathrm{V}}$ and $\rho_{\mathrm{H}_{2} \mathrm{O}}$ are modelled with Arrhenius type rates of the form

$$
\begin{aligned}
& \frac{d \rho_{\mathrm{V}}}{d t}=-B_{4} e^{-E_{4} / R T_{p}} \rho_{\mathrm{V}}, \\
& \frac{d \rho_{\mathrm{H}_{2} \mathrm{O}}}{d t}=-B_{5} e^{-E_{5} / R T_{p}} \rho_{\mathrm{H}_{2} \mathrm{O}} .
\end{aligned}
$$

These equations are based on the assumption that the particle heat conductivity is large to ensure that the temperature within the particle is nearly uniform, equal to $T_{p}$, then in this stage $\rho_{\mathrm{V}}$ and $\rho_{\mathrm{H}_{2} \mathrm{O}}$ are also nearly uniform.

Then the nondimensional particle gasification rates $\lambda_{4}$ and $\lambda_{5}$, defined as the mass rates of volatiles and humidity within the particles by the gasification reactions (4) and (5), divided by the characteristic diffusion flux $4 \pi a^{2} \rho_{g} \mathcal{D} / a$, are

$$
\begin{aligned}
& \lambda_{4}=\frac{a^{2}}{3 \rho_{g} \mathcal{D}} B_{4} e^{-E_{4} / \mathcal{R} T_{p}} \rho_{\mathrm{V}}, \\
& \lambda_{5}=\frac{a^{2}}{3 \rho_{g} \mathcal{D}} B_{5} e^{-E_{5} / \mathcal{R} T_{p}} \rho_{\mathrm{H}_{2} \mathrm{O}} .
\end{aligned}
$$

In a similar way, the nondimensional particle gasification rates $\lambda_{i}$, $i=1-3$, are defined as the mass rates of char consumption by the reaction $i$ divided by $4 \pi a \rho_{\mathrm{g}} \mathcal{D}$. The mass rate of gas generated within the particle divided by $4 \pi a \rho_{g} \mathcal{D}$, and denoted by $\lambda$, verifies $\lambda=\lambda_{1}+\lambda_{2}+\lambda_{3}+\lambda_{4}+\lambda_{5}$.

We cannot write a similar simple expression for the contributions, $\lambda_{1}, \lambda_{2}$ and $\lambda_{3}$, to the particle gas generation due to the char gasification reactions (1)-(3), because they depend on $Y_{\mathrm{CO}}, Y_{\mathrm{O}_{2}}$ and $Y_{\mathrm{H}_{2} \mathrm{O}}$ which are not uniform within the particle. However, we have developed in [11] a simplified treatment to describe the effects of the char gasification reactions, considering that the overall activation energies of these reactions are large. Then, for each of the reactions we can introduce a transition temperature, $T_{c}$, for which the Damkhöler number, defined as the ratio of the characteristic times of diffusion to the center of the particle and reaction, is equal to unity. Then, if the activation energy is large enough we can neglect the gasification reaction if the particle temperature $T_{p}$ is lower than (not very close to) $T_{c}$. This will be the case in the early stage of the particle history, but also, as occurs in the experiments analyzed below, in a later stage of the particle history when it is cooled below $T_{c}$ and, then, the gasification reaction is quenched. On the other hand, when the particle temperature $T_{p}$ raises above 
$T_{c}$ the reaction becomes so fast that it occurs, in a diffusion-controlled way, in a thin layer surrounding a shrinking char core where the mass fraction of the species gasifying the char is small compared to the unity.

The results of this double limit $\left(E_{i}\right.$ and $B_{i}$ tend to infinity with $T_{c}$ fixed) large activation energy analysis of the gasification reaction depend only on $T_{c}$, as a characteristic of the coal, and not on the values of the frequency factor or on the activation energy of the overall char gasification reactions, which depend on the coal type and are not well-known. In the numerical simulation given below, we have introduced the additional simplification that $T_{c}$ is the same for the three char gasification reactions, selecting $1100 \mathrm{~K}$ as the value of $T_{c}$ that better simulates the experimental results.

We shall give below the contributions of the char gasification reactions to the overall particle gasification rates in two limiting simplified models, referred in the following as BFLs1 and BFLs2. In both models the particles are considered to be small enough that, when they are in $\Omega_{O}$, they cannot sustain a diffusion flame around. In BFLs1 the initial particle content of ashes is enough to maintain a structurally stable surrounding shell, after the char is gasified in a thin layer, with fixed outer radius. BFLs2 corresponds to the case, typically considered in the literature, where the initial ash content of the particles is low so that the ash layer is disrupted after the char gasification. For the sake of completion we will also refer to the model BFL for the general case in [11].

Finally, when writing the energy equation for the particle, we consider the temperature to be spatially uniform and then it is given by the equation

$$
\begin{aligned}
\frac{4}{3} \pi a^{3} \rho_{p} c_{s} \frac{d T_{p}}{d t}= & 4 \pi a^{2}\left(q_{p}^{\prime \prime}+q_{r}^{\prime \prime}\right) \\
& +\int_{0}^{a}\left(\sum_{i=1}^{8} q_{i} \frac{3}{a^{2}} \rho_{g} \mathcal{D} \lambda_{i}\right) 4 \pi r^{2} d r,
\end{aligned}
$$

where $4 \pi a^{2} q_{p}^{\prime \prime}$ and $4 \pi a^{2} q_{r}^{\prime \prime}$ are the heat fluxes reaching the particle surface by conduction and radiation, given respectively by the relations

$q_{p}^{\prime \prime}=k \frac{d T}{d r_{r_{-} a^{+}}}, \quad q_{r}^{\prime \prime}=\varepsilon_{p}\left(\frac{1}{4} \int_{S^{2}} I(x, \omega) d \omega-\sigma T_{p}^{4}\right)$.

$I(x, \omega)$ is the radiation intensity in the direction $\omega$ at the position of the particle, denoted by $x, \varepsilon_{p}$ is the particle emissivity and $S^{2}$ is the unit sphere.

\subsubsection{First stage $\left(T_{p}<T_{c}\right)$ : frozen char gasification reactions}

In this stage the char gasification reactions can be considered frozen, so there is no production of $\mathrm{CO}$ or $\mathrm{H}_{2}$ within the particle. The energy equation for the evolution of $T_{p}$ in models BFLs 1 and BFLs2 (for the energy equation in the general BFL model see [11]) simplifies to

$\frac{4}{3} \pi a^{3} \rho_{p} c_{s} \frac{d T_{p}}{d t}=4 \pi a^{2}\left(q_{p}^{\prime \prime}+q_{r}^{\prime \prime}\right)+4 \pi \rho_{g} a \mathcal{D}\left(q_{4} \lambda_{4}+q_{5} \lambda_{5}\right)$,

where $q_{p}^{\prime \prime}$ is given by

$q_{p}^{\prime \prime}=\frac{k}{a c_{p}}\left(h_{T}^{g}-h_{T}^{s}\right) \frac{\lambda}{e^{\lambda}-1}$.

Here $\lambda=\lambda_{4}+\lambda_{5}$ is the nondimensional mass gasification rate of the particle, with the contribution $\lambda_{4}$ and $\lambda_{5}$ given by (18) and (19). The factor $\lambda /\left(e^{\lambda}-1\right)$, appearing in (23), is a Nusselt number accounting for the blocking effect to conduction heat transfer due to the Stefan radial flow associated with the gasification. We have neglected the effect of the convection associated to the relative motion of the particle and the local gas environment, considering the corresponding Reynolds number to be smaller than unity, as required for the validity of (23).
The mass of the particle, $\left(\rho_{\mathrm{V}}+\rho_{\mathrm{H}_{2} \mathrm{O}}+\rho_{C}+\rho_{a s h}\right) 4 \pi a^{3} / 3$, is changing with time in this stage (where $\rho_{C}$ and $\rho_{\text {ash }}$ do not change) due to changes in $\rho_{\mathrm{V}}$ and $\rho_{\mathrm{H}_{2} \mathrm{O}}$ given by (16) and (17).

2.2.2. Second stage $\left(T_{p}>T_{c}\right)$ : diffusion-controlled gasification reactions In this stage, the char gasification reactions are very fast (implying that $\mathrm{CO}_{2}, \mathrm{O}_{2}$ and $\mathrm{H}_{2} \mathrm{O}$ cannot coexist with char) and thus occur, in a diffusion-controlled way, in the surface $r=r_{c}(t)$ of a shrinking char core (see [14]). Thus, $Y_{\mathrm{CO}_{2}}=Y_{\mathrm{O}_{2}}=Y_{\mathrm{H}_{2} \mathrm{O}}=0$ for $r \leqslant r_{c}$ and $\rho_{C}=0$ for $r>r_{C}$ (only ashes are present in the particle for $r$ in the interval $\left.\left(r_{c}, a\right]\right)$. The second case of interest appears when the radius of the particle changes along the time, for example when the content of ashes in the coal is small, leading to the fragmentation of the porous layer. Then the gasification reactions will occur at $r=\delta_{p}$, the radius of the particle at time $t$. A detailed analysis of this situation was made by Gavalas [15].

The equations that we have to solve in the second combustion stage are different depending on the domain where the particle is, and on the three cases considered. In all these cases, the evolution of volatiles and moisture inside the coal particle will be evaluated using (16) and (17).

BFL: Large particles with high ash content, and gas oxidation reactions fast enough to sustain diffusion flames around or inside the particles (see [11]).

BFLs1: Small particles where a surrounding diffusion flame cannot be sustained, having sufficient ash content to maintain a non-swelling shell of ashes. This case has been advanced in [11] but the model was not fully developed there.

1. Particle in $\Omega_{F}$. In this case there is no oxygen in the neighborhood of the particle, only reactions (1) and (3) contribute to the char gasification. The resulting relations are

$$
\begin{aligned}
& \frac{11}{3} \frac{\lambda_{1}}{\lambda}=\left\{Y_{\mathrm{CO}_{2}}^{g}+\frac{11}{3} \frac{\lambda_{1}}{\lambda}\right\} e^{\lambda \frac{\mathcal{D}}{\mathcal{D}_{e}}\left(1-\frac{\alpha}{r_{c}}\right)-\lambda}, \\
& \frac{3}{2} \frac{\lambda_{3}}{\lambda}-\frac{\lambda_{5}}{\lambda}=\left\{Y_{\mathrm{H}_{2} \mathrm{O}}^{g}+\frac{3}{2} \frac{\lambda_{3}}{\lambda}-\frac{\lambda_{5}}{\lambda}\right\} e^{\lambda \frac{D}{\mathcal{D}_{\mathrm{e}}}\left(1-\frac{\alpha}{r_{c}}\right)-\lambda}, \\
& \frac{\rho_{c}^{0}}{\rho_{g} a D} r_{c}^{2} \frac{d r_{c}}{d t}=-\left(\lambda_{1}+\lambda_{3}\right) .
\end{aligned}
$$

The particle temperature is determined by

$$
\begin{aligned}
\frac{4}{3} \pi a^{3} \rho_{p} c_{s} \frac{d T_{p}}{d t}= & 4 \pi a^{2}\left(q_{p}^{\prime \prime}+q_{r}^{\prime \prime}\right)+4 \pi \rho_{g} a \mathcal{D}\left(q_{1} \lambda_{1}\right. \\
& \left.+q_{3} \lambda_{3}+q_{4} \lambda_{4}+q_{5} \lambda_{5}\right)
\end{aligned}
$$

where $q_{p}^{\prime \prime}$ is given by (23) and $q_{r}^{\prime \prime}$ by (21).

2. Particle in $\Omega_{\mathrm{O}}$. In this case species $\mathrm{CO}_{2}, \mathrm{O}_{2}$ and $\mathrm{H}_{2} \mathrm{O}$ contribute to the char gasification. We thus obtain the relations

$$
\begin{aligned}
& \frac{11}{3} \frac{\lambda_{1}}{\lambda}=\left\{Y_{\mathrm{CO}_{2}}^{\mathrm{g}}+\frac{11}{3} \frac{\lambda_{1}}{\lambda}\right\} e^{\lambda \frac{D}{D_{e}}\left(1-\frac{a}{r_{c}}\right)-\lambda}, \\
& \frac{4}{3} \frac{\lambda_{2}}{\lambda}=\left\{Y_{\mathrm{O}_{2}}^{g}+\frac{4}{3} \frac{\lambda_{2}}{\lambda}\right\} e^{\lambda \frac{\mathcal{D}}{D_{e}}\left(1-\frac{a}{r_{c}}\right)-\lambda}, \\
& \frac{3}{2} \frac{\lambda_{3}}{\lambda}-\frac{\lambda_{5}}{\lambda}=\left\{Y_{\mathrm{H}_{2} \mathrm{O}}^{g}+\frac{3}{2} \frac{\lambda_{3}}{\lambda}-\frac{\lambda_{5}}{\lambda}\right\} e^{\lambda \frac{\mathcal{D}}{D_{e}}\left(1-\frac{a}{r_{c}}\right)-\lambda}, \\
& \frac{\rho_{C}^{0}}{\rho_{g} a D} r_{c}^{2} \frac{d r_{c}}{d t}=-\left(\lambda_{1}+\lambda_{2}+\lambda_{3}\right) .
\end{aligned}
$$

The particle temperature evolution is given by

$$
\begin{aligned}
\frac{4}{3} \pi a^{3} \rho_{p} c_{s} \frac{d T_{p}}{d t}= & 4 \pi a^{2}\left(q_{p}^{\prime \prime}+q_{r}^{\prime \prime}\right)+4 \pi \rho_{g} a \mathcal{D}\left(q_{1} \lambda_{1}\right. \\
& \left.+q_{2} \lambda_{2}+q_{3} \lambda_{3}+q_{4} \lambda_{4}+q_{5} \lambda_{5}\right)
\end{aligned}
$$

with $q_{p}^{\prime \prime}$ and $q_{r}^{\prime \prime}$ given by (23) and (21).

BFLs2: Particle with low ash content, with disrupted ash layer and thus with variable radius $\delta_{p}$. This case was not considered in [11]. By computations similar to those in [11], we obtain the following relations: 
1. Particle in $\Omega_{F}$. In this case there is no oxygen in the neighborhood of the particle, only reactions (1) and (3) contribute to the char gasification. The resulting relations are

$$
\begin{aligned}
& \frac{11}{3} \frac{\lambda_{1}}{\lambda}=\left\{Y_{\mathrm{CO}_{2}}^{\mathrm{g}}+\frac{11}{3} \frac{\lambda_{1}}{\lambda}\right\} e^{-\lambda}, \\
& \frac{3}{2} \frac{\lambda_{3}}{\lambda}-\frac{\lambda_{5}}{\lambda}=\left\{Y_{\mathrm{H}_{2} \mathrm{O}}^{\mathrm{g}}+\frac{3}{2} \frac{\lambda_{3}}{\lambda}-\frac{\lambda_{5}}{\lambda}\right\} e^{-\lambda}, \\
& \frac{\rho_{C}^{0}}{\rho_{\mathrm{g}} \mathrm{D}} \delta_{p} \frac{d \delta_{p}}{d t}=-\left(\lambda_{1}+\lambda_{3}\right) .
\end{aligned}
$$

The particle temperature evolution is given by

$$
\begin{aligned}
\frac{4}{3} \pi \delta_{p}^{3} \rho_{p} c_{s} \frac{d T_{p}}{d t}= & 4 \pi \delta_{p}^{2}\left(q_{p}^{\prime \prime}+q_{r}^{\prime \prime}\right)+4 \pi \rho_{g} \delta_{p} \mathcal{D}\left(q_{1} \lambda_{1}\right. \\
& \left.+q_{3} \lambda_{3}+q_{4} \lambda_{4}+q_{5} \lambda_{5}\right)
\end{aligned}
$$

where $q_{p}^{\prime \prime}$ is given by (23) and $q_{r}^{\prime \prime}$ by (21).

2. Particle in $\Omega_{\mathrm{O}}$. In this case $\mathrm{CO}_{2}, \mathrm{O}_{2}$ and $\mathrm{H}_{2} \mathrm{O}$ contribute to the char gasification, with the result

$$
\left.\begin{array}{l}
\frac{11}{3} \frac{\lambda_{1}}{\lambda}=\left\{Y_{\mathrm{CO}_{2}}^{\mathrm{g}}+\frac{11}{3} \frac{\lambda_{1}}{\lambda}\right\} e^{-\lambda}, \\
\frac{4}{3} \frac{\lambda_{2}}{\lambda}=\left\{Y_{\mathrm{O}_{2}}^{\mathrm{g}}+\frac{4}{3} \frac{\lambda_{2}}{\lambda}\right\} e^{-\lambda}, \\
\frac{3}{2} \frac{\lambda_{3}}{\lambda}-\frac{\lambda_{5}}{\lambda}=\left\{Y_{\mathrm{H}_{2} \mathrm{O}}^{\mathrm{g}}+\frac{3}{2} \frac{\lambda_{3}}{\lambda}-\frac{\lambda_{5}}{\lambda}\right\} e^{-\lambda}, \\
\frac{\rho_{C}^{0}}{\rho_{\mathrm{g}} \mathcal{D}} \delta_{p} \frac{d \delta_{p}}{d t}=-\left(\lambda_{1}+\lambda_{2}+\lambda_{3}\right) .
\end{array}\right]
$$

The particle temperature has to be determined from the equation

$$
\begin{aligned}
\frac{4}{3} \pi \delta_{p}^{3} \rho_{p} c_{s} \frac{d T_{p}}{d t}= & 4 \pi \delta_{p}^{2}\left(q_{p}^{\prime \prime}+q_{r}^{\prime \prime}\right)+4 \pi \rho_{g} \delta_{p} \mathcal{D}\left(q_{1} \lambda_{1}+q_{2} \lambda_{2}\right. \\
& \left.+q_{3} \lambda_{3}+q_{4} \lambda_{4}+q_{5} \lambda_{5}\right)
\end{aligned}
$$

where $q_{p}^{\prime \prime}$ and $q_{r}^{\prime \prime}$ are given again by (23) and (21).

\subsection{Homogenized sources to the gas phase}

One of the purposes of solving the solid phase model is to obtain the sources of mass and energy to the gas phase from the gasification of the coal particles. Once we know these sources we can solve Eqs. (3)-(7) and obtain the temperature and composition of the gas mixture.

The homogenized sources in the gas phase per unit volume and time, at point $x$, are computed adding the contributions of each single particle that at instant $t$ is at position $x$ by the expression

$f^{\alpha}(\mathbf{x})=\sum_{j=1}^{N_{e}} \sum_{i=1}^{N_{p}} \tilde{q}_{j} \frac{p_{i j}}{100} \int_{0}^{t_{f}^{i j}} F_{i j}^{\alpha}(t) \delta\left(\mathbf{x}-\mathbf{x}_{p}^{i j}(t)\right) d t$,

where $F_{i j}^{\alpha}(t)$ is the source of mass or energy of one particle of type $i$ introduced through inlet $j$ at instant $t, \mathbf{x}_{p}^{i j}(t)$ is the position occupied by this particle at instant $t, \delta(x)$ is the Dirac measure at point $0, t_{f}^{i j}$ is the time needed for the particle to be completely burned or to leave the furnace, $\vec{q}_{j}$ is the mass flow of coal through the inlet $j, p_{i j}$ is the percentage of particles of type $i$ through inlet $j$, and $N_{e}$ and $N_{p}$ are the number of inlets and types of particles, respectively.

The expressions for the sources of mass of each of the species due to one single particle are the following:

BFL: Particle with large ash content, able to sustain a surrounding diffusion flame (see again [11]).

BFLs1: Particle is small but its size does not change during combustion.

1. In $\Omega_{\mathrm{F}}\left(Y_{\mathrm{O}_{2}}^{g}=0\right)$ :

$F_{\mathrm{O}_{2}}^{\mathrm{m}}=F_{\mathrm{SO}_{2}}^{\mathrm{m}}=0$,

$F_{\mathrm{CO}_{2}}^{\mathrm{m}}=\frac{4 \pi a k}{c_{p}}\left(-\frac{11}{3} \lambda_{1}\right)$,

$$
\begin{aligned}
& F_{\mathrm{H}_{2} \mathrm{O}}^{m}=\frac{4 \pi a k}{c_{p}}\left(\lambda_{5}-\frac{3}{2} \lambda_{3}\right), \\
& F_{\mathrm{CO}}^{m}=\frac{4 \pi a k}{c_{p}}\left(\frac{14}{3} \lambda_{1}+\frac{7}{3} \lambda_{3}\right), \\
& F_{\mathrm{V}}^{m}=\frac{4 \pi a k}{c_{p}} \lambda_{4}, \\
& F_{\mathrm{H}_{2}}^{m}=\frac{4 \pi a k}{c_{p}} \frac{1}{6} \lambda_{3} . \\
& 2 . \quad \text { In } \Omega_{0}\left(Y_{\mathrm{O}_{2}}^{g}>0\right): \\
& F_{\mathrm{O}_{2}}^{m}=\frac{4 \pi a k}{c_{p}}\left(-\frac{4}{3} \lambda_{2}\right), \\
& F_{\mathrm{CO}_{2}}^{m}=\frac{4 \pi a k}{c_{p}}\left(-\frac{11}{3} \lambda_{1}\right), \\
& F_{\mathrm{H}_{2} \mathrm{O}}^{m}=\frac{4 \pi a k}{c_{p}}\left(\lambda_{5}-\frac{3}{2} \lambda_{3}\right), \\
& F_{\mathrm{SO}_{2}}^{m}=0, \\
& F_{\mathrm{CO}}^{m}=\frac{4 \pi a k}{c_{p}}\left(\frac{14}{3} \lambda_{1}+\frac{7}{3} \lambda_{2}+\frac{7}{3} \lambda_{3}\right), \\
& F_{\mathrm{V}}^{m}=\frac{4 \pi a k}{c_{p}} \lambda_{4}, \\
& F_{\mathrm{H}_{2}}^{m}=\frac{4 \pi a k}{c_{p}} \frac{1}{6} \lambda_{3} .
\end{aligned}
$$

BFLs2: Particle size changes due to the low ash content.

1. In $\Omega_{\mathrm{F}}\left(Y_{\mathrm{O}_{2}}^{\mathrm{g}}=0\right)$ :

$$
\begin{aligned}
& F_{\mathrm{O}_{2}}^{m}=F_{\mathrm{SO}_{2}}^{m}=0 \text {, } \\
& F_{\mathrm{CO}_{2}}^{m}=\frac{4 \pi \delta_{p} k}{c_{p}}\left(-\frac{11}{3} \lambda_{1}\right) \text {, } \\
& F_{\mathrm{H}_{2} \mathrm{O}}^{m}=\frac{4 \pi \delta_{p} k}{c_{p}}\left(\lambda_{5}-\frac{3}{2} \lambda_{3}\right) \text {, } \\
& F_{\mathrm{CO}}^{m}=\frac{4 \pi \delta_{p} k}{c_{p}}\left(\frac{14}{3} \lambda_{1}+\frac{7}{3} \lambda_{3}\right), \\
& F_{\mathrm{V}}^{m}=\frac{4 \pi \delta_{p} k}{c_{p}} \lambda_{4} \\
& F_{\mathrm{H}_{2}}^{m}=\frac{4 \pi \delta_{p} k}{c_{p}} \frac{1}{6} \lambda_{3} . \\
& \text { 2. In } \Omega_{0}\left(\mathrm{Y}_{\mathrm{O}_{2}}^{g}>0\right) \text { : } \\
& F_{\mathrm{O}_{2}}^{m}=\frac{4 \pi \delta_{p} k}{c_{p}}\left(-\frac{4}{3} \lambda_{2}\right), \\
& F_{\mathrm{CO}_{2}}^{m}=\frac{4 \pi \delta_{p} k}{c_{p}}\left(-\frac{11}{3} \lambda_{1}\right) \text {, } \\
& F_{\mathrm{H}_{2} \mathrm{O}}^{m}=\frac{4 \pi \delta_{p} k}{c_{p}}\left(\lambda_{5}-\frac{3}{2} \lambda_{3}\right) \text {, } \\
& F_{\mathrm{SO}_{2}}^{m}=0 \text {, } \\
& F_{\mathrm{CO}}^{m}=\frac{4 \pi \delta_{p} k}{c_{p}}\left(\frac{14}{3} \lambda_{1}+\frac{7}{3} \lambda_{2}+\frac{7}{3} \lambda_{3}\right), \\
& F_{\mathrm{V}}^{m}=\frac{4 \pi \delta_{p} k}{c_{p}} \lambda_{4}, \\
& F_{\mathrm{H}_{2}}^{m}=\frac{4 \pi \delta_{p} k}{c_{p}} \frac{1}{6} \lambda_{3} \text {. }
\end{aligned}
$$

In every region of the domain the total sources of mass and energy for the BFLs1 model are

$F^{m}=\frac{4 \pi a k}{c_{p}} \lambda$, 
$F^{e}=4 \pi a k\left(\frac{c_{S}}{c_{p}} T_{p}-T_{g}\right) \frac{\lambda}{e^{\lambda}-1}-c_{S} T_{p} \frac{d m_{p}}{d t}$,

whereas for the BFLs2 model they are

$F^{m}=\frac{4 \pi \delta_{p} k}{c_{p}} \lambda$,

$F^{e}=4 \pi \delta_{p} k\left(\frac{c_{s}}{c_{p}} T_{p}-T_{g}\right) \frac{\lambda}{e^{\lambda}-1}-c_{s} T_{p} \frac{d m_{p}}{d t}$.

\section{Particle motion model}

Since a Lagrangian description for the analysis of the coal particles behavior is used, the trajectory of each single particle throughout the computational domain has to be calculated. The particle motion model takes into account the drag and gravity forces acting on the particles. Therefore, the particle velocity can be obtained by solving the initial value problem:

$\frac{d \mathbf{v}_{p}}{d t}=F_{A}\left(\mathbf{v}_{g}-\mathbf{v}_{p}\right)+\mathbf{g}$,

$\mathbf{v}_{p}(0)=\mathbf{v}_{p 0}$,

where $F_{A}\left(\mathbf{v}_{g}-\mathbf{v}_{p}\right)$ is the drag force per unit mass and

$F_{A}=\frac{3}{16} \frac{\mu}{\rho_{p} a^{2}} C_{D} R e$.

Here $\mathbf{v}_{g}$ is the gas mixture velocity, $\mathbf{v}_{p}$ the particle velocity, $\mu$ the gas viscosity, $\operatorname{Re}$ is the Reynolds number relative to the particle,

$\operatorname{Re}=\rho_{g}\left\|\mathbf{v}_{g}-\mathbf{v}_{p}\right\| \frac{2 a}{\mu}$,

and $C_{D}$ is the drag coefficient written in an approximate form as (see Wallis [16])

$C_{D}= \begin{cases}\frac{1+0.15 R^{0.687}}{R e / 24} & \text { if } R e \leqslant 1000 \\ 0.44 & \text { otherwise. }\end{cases}$

This drag force does not include a small reduction associated to the blowing effect due to gasification; this reduction is compensated by an increase in the drag due to the effect of the variable density and viscosity of the gas in the neighborhood of the particle.

\subsection{Stochastic particle dispersion modeling}

The particle motion model given by (59-63) needs the instantaneous local value of the velocity of the gas mixture. This value can be obtained by coupling this particle motion model with a stochastic model to take into account the particle dispersion; for example, a discrete random walk model. This kind of models computes the particle dispersion with random sampling to obtain instantaneous flow properties for a statistically significant number of particle trajectories. Each trajectory calculated for each particle size and initial location is called a "try". The selected model computes the instantaneous velocity as the sum of the mean fluid velocity and a Gaussian distributed random velocity fluctuation with zero mean and variance related to the turbulent velocity scale computed from the used turbulence model.

In the present paper we use the standard $k-\epsilon$ model to estimate the turbulence properties. Thus the instantaneous fluid velocity is calculated as

$\mathbf{v}_{g}=\overline{\mathbf{v}}_{g}+\left(\xi_{1} \sqrt{\frac{2 k}{3}}, \xi_{2} \sqrt{\frac{2 k}{3}}, \xi_{3} \sqrt{\frac{2 k}{3}}\right)$

where $\xi_{i}, i=1-3$, are normally distributed random numbers. The eddy lifetime is expressed as $\tau_{e}=-0.15 \frac{k}{\epsilon} \log (r)$

where $r$ is a uniformly distributed random number between 0 and 1 , which leads to a more realistic description of the correlation function (see Tian and Ahmadi [17]). The time required for the particle to cross an eddy is given by

$\tau_{c}=-\tau_{p} \log \left(1-\frac{l_{e}}{\tau_{p}\left\|\mathbf{v}_{g}-\mathbf{v}_{p}\right\|}\right)$,

where

$\tau_{p}=\frac{4 \rho_{p} a^{2}}{18 \mu}$

is the Stokes particle relaxation time, and

$l_{e}=0.09 \frac{k^{1.5}}{\epsilon}$

is the eddy length scale. Thus, when the minimum of $\tau_{e}$ and $\tau_{c}$ is elapsed a new velocity fluctuation has to be calculated. This model is based on the eddy-particle interaction model introduced by Gosman and Ioannides [18]. As many random walk models, this model produces reasonably good behavior for flows with homogeneous turbulence. In [19], for example, the effect of anisotropy has been included. The suitability of many others random walk models and particle dispersion models was analyzed in $[20,21]$.

\section{Numerical solution}

While in the previous sections we have presented the combustion models, in this section we will briefly introduce the algorithms for their numerical solution. We will also show some numerical results obtained for an experimental coal flame.

\subsection{Algorithm for the particle model}

Before solving the solid phase model, the velocity, turbulence parameters, composition and temperature of the gas mixture have to be computed. In particular, mass fractions and temperature can be obtained using the gas phase model summarized in Section 2.1.

For the solid phase model, we have developed an algorithm based on a Lagrangian description. We follow the trajectory of many particles chosen according to the size distribution and solve the corresponding equations for each of them step by step. Therefore, this algorithm is characterized by its ability to distinguish the combustion stage each particle undergoes at any time. For each particle and time step, the iterative scheme for the BFLs1 model is as follows:

1. Compute the particle velocity $\mathbf{v}_{p}^{n}$ and, as a consequence, its position $\mathbf{x}_{p}^{n}$.

2. Interpolate mass fractions $Y_{\mathrm{O}_{2}}^{g}, Y_{\mathrm{CO}_{2}}^{g}$ and $Y_{\mathrm{H}_{2} \mathrm{O}}^{\mathrm{g}}$, temperature $T_{\mathrm{g}}$, and turbulence parameters $k$ and $\epsilon$ of the gas mixture at position $\mathbf{x}_{p}^{n}$ occupied by the particle. To know the element of the mesh where the particle is we use the search-locate algorithm developed by Allievi and Bermejo [22]. This fast and efficient method allows us to use non-structured meshes. Furthermore, its implementation is particularly simple in finite element codes since it uses concepts that are standard in this methodology.

3. Solve (16) and (17).

4. If $T_{p}<T_{c}$ (char gasification reactions are frozen) go to 7 .

5. If $Y_{\mathrm{O}_{2}}^{g}=0$ obtain $\lambda_{1}, \lambda_{3}$ and $r_{c}$ by solving (D1) and go to 7 .

6. If $Y_{\mathrm{O}_{2}}^{\mathrm{g}}>0$ obtain $\lambda_{1}, \lambda_{2}, \lambda_{3}$ and $r_{c}$ by solving (D2) and go to 7 .

7. Solve the energy equation and obtain the particle temperature. 
8. Calculate the contribution of the particle to the homogenized sources of mass and energy.

9. If the particle exits the domain, calculations have finished. otherwise go to 1 .

A scheme of the above algorithm, which has been advanced by Bermúdez et al. [23] for the BFL version of the model, can be seen in Fig. 2.

\subsection{Numerical methods}

The discrete phase model equations are easy to solve and do not need sophisticated numerical methods. For this reason, the nonlinear algebraic systems appearing in the model are solved using a discrete Newton method and the ordinary differential equations are solved with the explicit Euler method.

Recall that the gas phase model involves convection-diffusionreaction equations. This kind of equations can be written as

$$
\begin{aligned}
& \rho_{g}(x, t) \frac{D \boldsymbol{u}(x, t)}{D t}-\nabla \cdot\left(\rho_{g}(x, t) \mathcal{D}(x) \nabla \boldsymbol{u}(x, t)\right)+f^{\mathrm{m}}(x, t) \boldsymbol{u}(x, t) \\
& \quad=f(x, t),(x, t) \in \Omega \times(0, T),
\end{aligned}
$$

where $u$ is any of the Shvab-Zeldovich variables (8)-(12). Discretization of these equations has been done by using a semi-Lagrangian
Galerkin method. For time discretization we have used a Backward Difference Formula of order 2 (BDF2). Specifically, the material time derivative has been discretized using the scheme

$$
\frac{D u}{D t}=\frac{\partial u}{\partial t}+\mathbf{v}_{g} \cdot \nabla u \approx \frac{1}{2 \Delta t}\left(3 u^{n+1}-4 u^{n} \circ \chi_{1}^{n}+u^{n-1} \circ \chi_{2}^{n}\right)
$$

where

$$
\chi_{i}^{n}(x)=X\left(x, t^{n+1} ; t^{n+1}-i \Delta t\right), i=1,2,
$$

and $X\left(x, t^{n+1} ; t\right)$ is the position at time $t$ of the fluid particle that is at point $x$ at time $t^{n+1}$ and that moves with velocity $\mathbf{v}_{g}$.

\subsection{Pulverized coal jet flame simulation}

In order to test our combustion model we have simulated a pulverized coal jet flame experiment done by Hwang et al. [12,13], where a study of the structure of a turbulent pulverized coal jet flame has been carried out. The authors of these papers present many results obtained by optical measurements that help to clarify the processes involved in pulverized coal combustion. For the experiment, a laboratory scale burner was specially manufactured to obtain a pulverized coal jet burning with a flame in ambient air. This burner has a coaxial structure made up of a main burner and an annular slit burner. A scheme of the coal burner can be seen in

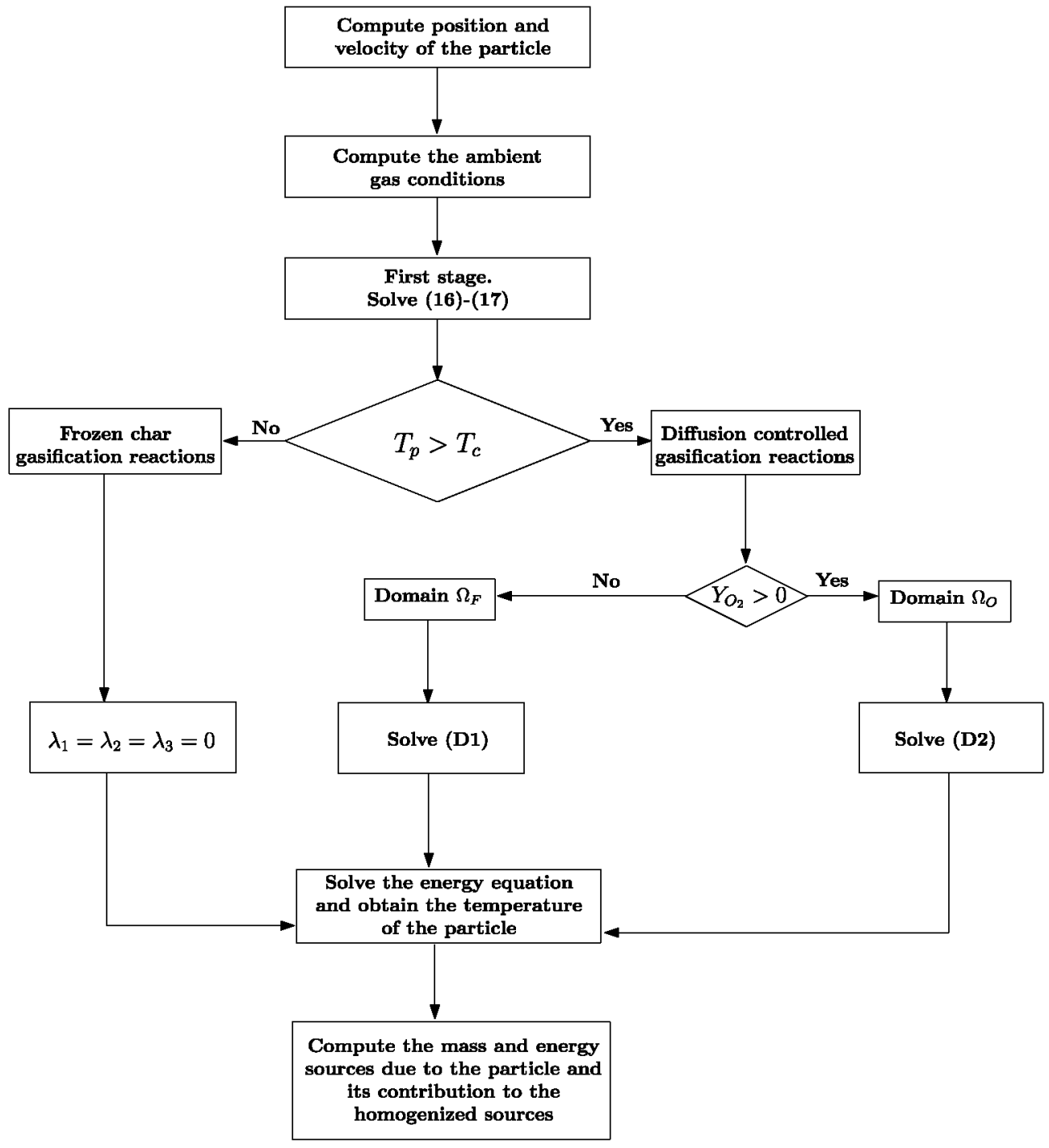

Fig. 2. Algorithm for solving the solid phase in the BFLs1 model. 
Fig. 3. In the experiment, methane is supplied to generate an annular pilot flame; this is also needed for the initial heating of the particles to provide the volatiles required for the flame stabilization of the pulverized jet in the non-heated ambient air. The methane flow

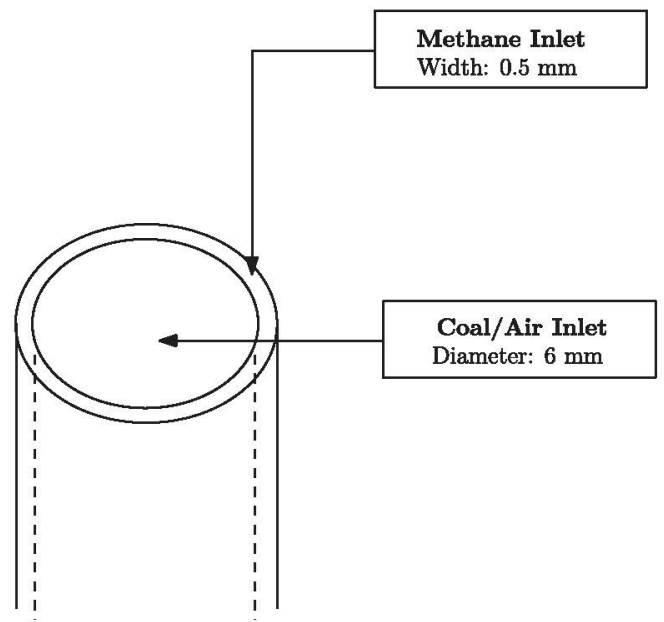

Fig. 3. Scheme of the pulverized coal burner.

Table 1

Experimental conditions.

\begin{tabular}{ll}
\hline Air flow rate & $1.80 \times 10^{-4} \mathrm{~m}^{3} / \mathrm{s}$ \\
$\mathrm{CH}_{4}$ flow rate & $2.33 \times 10^{-5} \mathrm{~m}^{3} / \mathrm{s}$ \\
Pulverized coal flow rate & $1.49 \times 10^{-4} \mathrm{~kg} / \mathrm{s}$ \\
\hline
\end{tabular}

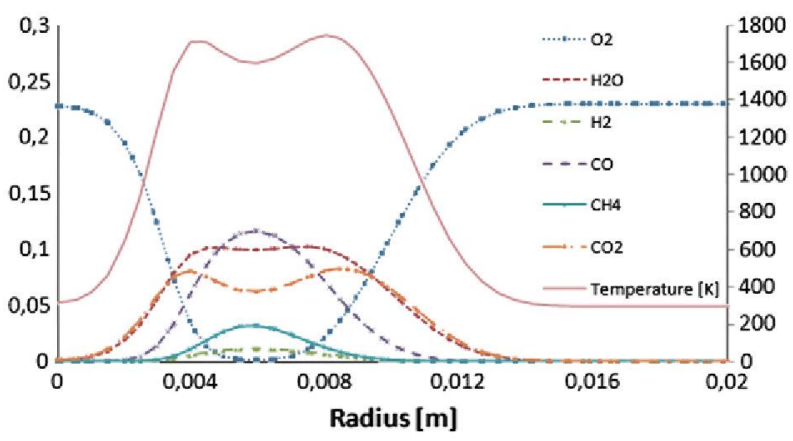

Fig. 4. Profiles of species mass fractions and temperature at $20 \mathrm{~mm}$ from the burner. rate is the minimum needed to form a stable flame. Firstly, the air is supplied to the main burner port and the methane to the annular slit burner. The gas flame due to the air and the methane is formed. When the methane diffusion flame becomes stable the pulverized coal particles are injected. In Table 1 some experimental conditions are specified.

We have chosen this experiment because of the difficulty of finding a flame in a situation where the flow is laminar and the numerical description of the problem is simple. In addition, we cannot use for the moment our model for the description of a turbulent jet, because we have not yet incorporated in our model a presumed PDF scheme to calculate, in a reliable way, the mean values of the temperature and species mass fractions in terms of the presumed PDF of the conserved scalars. As we said before, the stabilization of the flame has been achieved by an annular injection of methane (giving a pilot flame) in place of the more frequent (but also more complicated to simulate) generation of swirl in the injector.

On the other hand, it should be noticed that the experiment of Hwang et al. is not fully turbulent but transitional (in fact they indicate the Reynolds number to be of order 2500 based on the kinematic viscosity at ambient conditions, but with the typical temperatures reached in the flame it will be five times lower). This does not allow us to expect a good agreement between the experimental results and the numerical ones.

An axisymmetric steady-state simulation of the first stage of the experiment was performed with the commercial code FLUENT version 12.1.4. Turbulence was calculated using the standard $k-\epsilon$ model and for the species and temperature the mixture fraction method was used with a PDF table for the treatment of the turbulence-chemistry interaction. This simulation have been carried out to calculate profiles for the velocity, the temperature, the composition and the turbulence parameters of the gas mixture near the burner. The calculated profiles at $20 \mathrm{~mm}$ from the burner are plotted in Figs. 4 and 5. With these profiles we will carry out three 3D simulations with the different versions of our code corresponding to the three models given above. The purpose of these simulations is to compare our three models among them and with the experimental data.

The coal used in the experiment is Newlands bituminous coal. Proximate and ultimate analysis of this coal as well as the heating value, density and specific heat, provided by Hwang et al., are given in Table 2. For the simulations, we have used a size range of 5$61 \mu \mathrm{m}$ and a Rosin-Rammler distribution function to represent the experimental data. The mass percentage of particles for each diameter range is plotted in Fig. 6. The mean diameter of this distribution is $33.3 \mu \mathrm{m}$ and to fit the Rosin-Rammler curve to this data the value of the spread parameter has been taken equal to 4.02 .

We can consider, with little error, that all the particle moisture is evaporated before leaving the burner. The kinetic parameters for the devolatilization are $E_{4}=3.11 \times 10^{7} \mathrm{~J} / \mathrm{kgmol}$ and $B_{4}=2021 \mathrm{~s}^{-1}$. The critical particle temperature of transition in the rate of the char
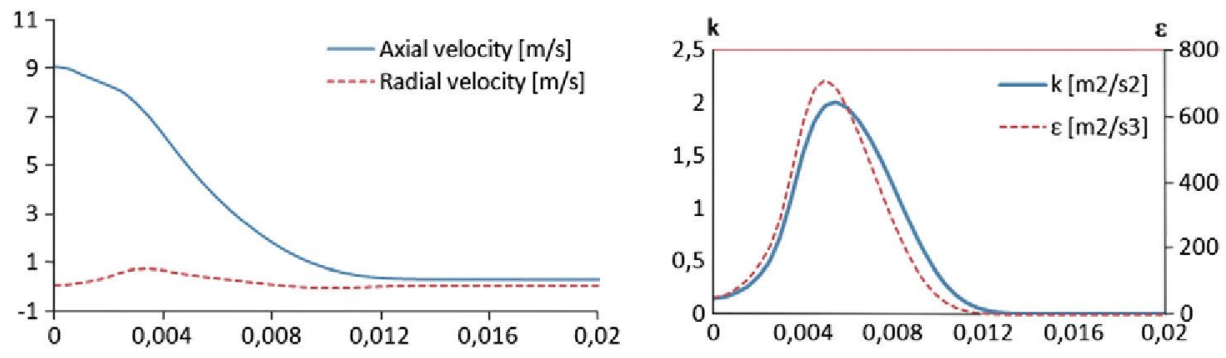

Fig. 5. Profiles of velocity, $k$ and $\epsilon$ at $20 \mathrm{~mm}$ from the burner. 
Table 2

Properties of coal.

\begin{tabular}{lc}
\hline Proximate analysis (Dry basis) (wt\%) \\
\hline Volatile matter & 26.9 \\
Fixed carbon & 57.9 \\
Ash & 15.2 \\
Ultimate analysis (Dry basis) (wt\%) \\
C & 71.9 \\
H & 4.4 \\
N & 1.58 \\
O & 6.53 \\
S & 0.39 \\
High heating value & $2.81 \times 10^{7} \mathrm{~J} / \mathrm{kg}$ \\
Density & $1000 \mathrm{~kg} / \mathrm{m}^{3}$ \\
Specific heat & $1000 \mathrm{~J} /(\mathrm{kg} \mathrm{K})$ \\
\hline
\end{tabular}

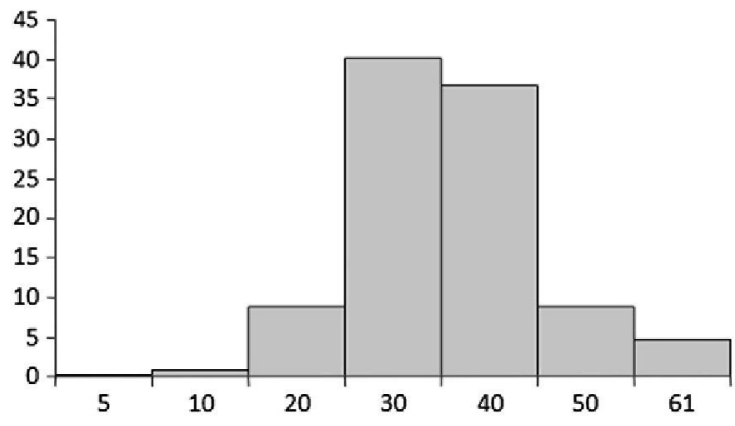

Fig. 6. Mass percentage of particles by diameter range.

gasification reactions of the particles has been chosen as $T_{C}=1100 \mathrm{~K}$. Then, when the particle temperature is lower than this critical value the three char gasification reactions are frozen, and when it is above $1100 \mathrm{~K}$ they are considered to be infinitely fast and diffusion-controlled. When, due to the cooling by the ambient air entrainment, the temperature of the particle drops below

Table 3

Results from the combustion models.

\begin{tabular}{lll}
\hline & Released volatiles (\%) & Gasified char (\%) \\
\hline BFL & 99.99 & 11.87 \\
BFLs1 & 99.99 & 11.51 \\
BFLs2 & 99.99 & 98.49 \\
\hline
\end{tabular}

$1100 \mathrm{~K}$, the char gasification reactions are quenched so that a significant fraction of the char remains unburned.

The gasified char mentioned in the experiment is $11.3 \%$ (all percentages are in terms of mass) and the emitted volatile matter is $44.6 \%$, less than half of the volatile matter assigned by the proximate analysis. The remaining volatiles join the ashes, thus very likely facilitating the structural stability of the ash shell. Anyway, our model should be extended to account for the presence of two types of volatiles with different rates of devolatilization. The percentage of released volatiles and gasified char provided by our models are shown in Table 3.

We shall begin now the comparison of the results of the three models by showing in Fig. 7 the cross section, by an axial plane, of the two surfaces of the two diffusion flames $\Gamma_{F}$ encountered in our models, that enclose two regions $\Omega_{F}$ without oxygen; the latter is prevented from entering these regions by the enveloping diffusion flames, and thus it is found only outside, in domain $\Omega_{O}$. One of these diffusion flames corresponds to the annular pilot methane flame; this is needed in the experiments, due to the absence of significant radiation effects, to initially heat the injected air. This air heats the particles to facilitate their devolatilization and the char gasification. The fast gas phase oxidation reactions of the volatiles, $\mathrm{H}_{2}$ and $\mathrm{CO}$ generated by the particle gasification, take place in two forms: either as diffusion-controlled group combustion in the second of the diffusion flames found downstream, or in the domain $\Omega_{O}$, in a distributed way in our models BFLs1 and BFLs 2 or in the form of closed diffusion flames, inside or in the neighborhood of the particles, in the BFL model. The homogenized gas phase concentration of the volatiles, $\mathrm{H}_{2}$ and $\mathrm{CO}$ is negligible in the domain $\Omega_{O}$; see, for example, the non-zero values of the mass fraction of CO, shown in Fig. 8 and also in Fig. 13, that are confined to the domains $\Omega_{F}$ bounded by the diffusion flame, with values of their mass fraction decreasing to zero when $\Gamma_{F}$ is approached.

In Fig. 9 we plot the homogenized sources of mass obtained with the three models. The mass fractions of $\mathrm{SO}_{2}$ obtained with the three models are shown in Fig. 10. The mass fraction of $\mathrm{SO}_{2}$ (one important pollutant) results in our models from the amount of $S$ provided by the ultimate analysis of the coal, which we incorporate to the volatile matter to be oxidized in the gas phase. The gas temperature obtained with our models is shown in Fig. 11, with a colored code and plotting some isotherms indicated in the color code. The irregularities in the flame surface and lack of axial symmetry are due to the numerical scheme. In Fig. 12 we plot the axial distribution of the gas temperature. Figure 13 shows the radial distribution, at $z=90 \mathrm{~mm}$, of the mass fraction of $\mathrm{O}_{2}$ and $\mathrm{CO}$ obtained with the BFLs1 model and the gas temperature obtained

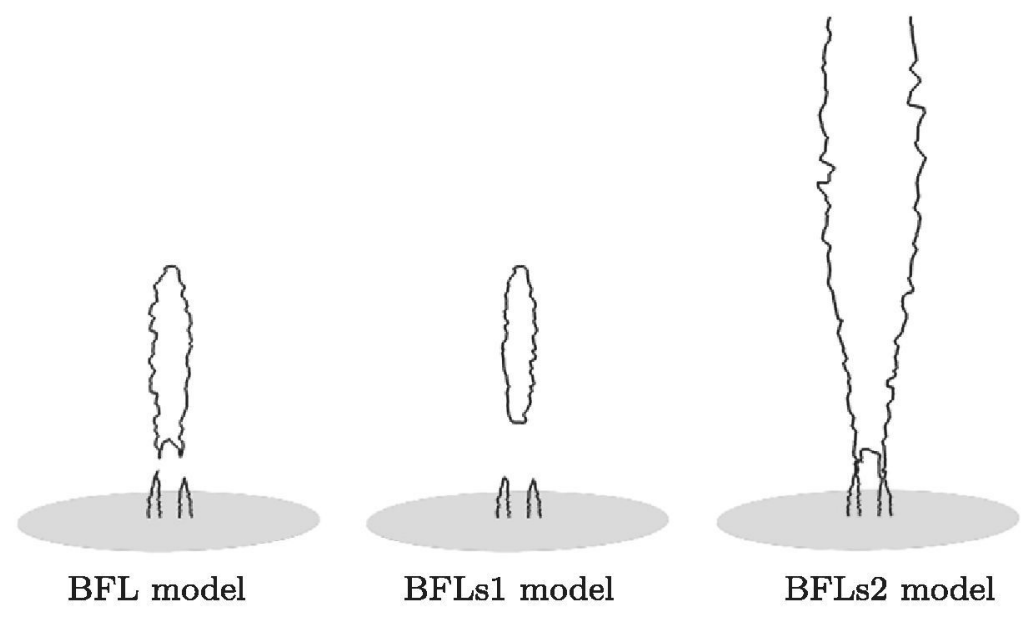

Fig. 7. Position of the diffusion flame. 


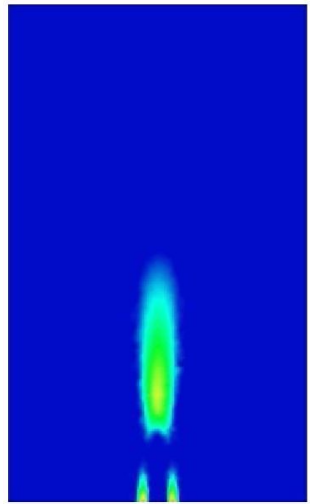

BFL model

monox
0.3000
0.2842
0.2684
0.2526
0.2368
0.2211
0.2053
0.1895
0.1737
0.1579
0.1421
0.1263
0.1105
0.0947
0.0789
0.0632
0.0474
0.0316
0.0158
0.0000

0.0000

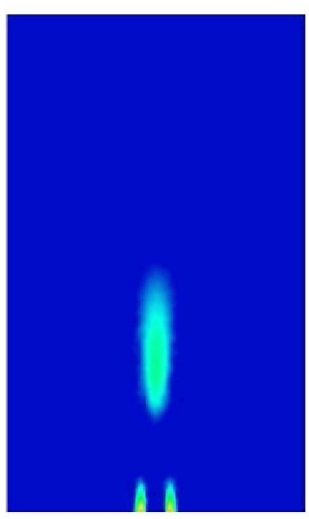

BFLs1 model

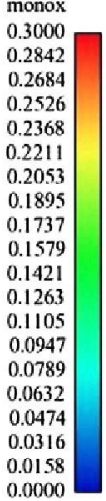

0.0000

Fig. 8. Mass fraction of CO.

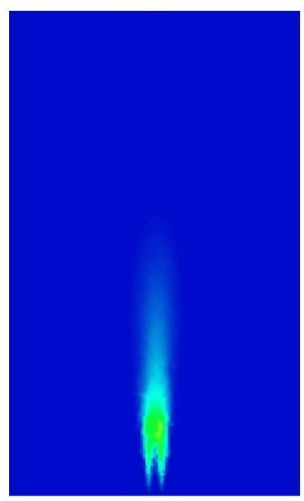

BFL model

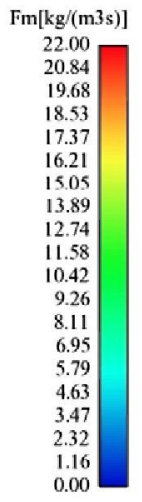

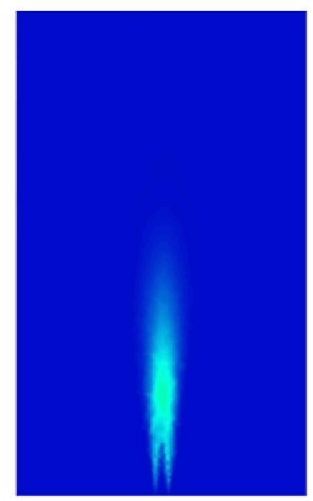

BFLs1 model

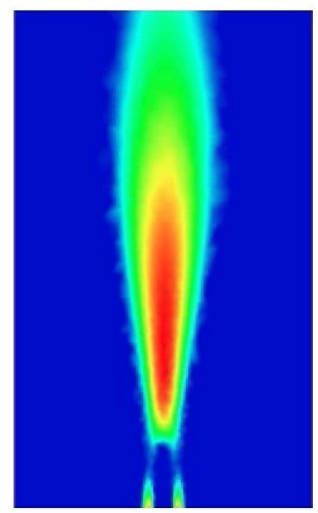

monox

0.3000

0.2842

0.2684

0.2526

0.2211

0.2053

0.1895

0.1737

0.1579

0.1421

0.1263

0.1105

0.0947

0.0789

0.0632

0.0474

0.0316

0.0158

BFLs2 model

0.0000

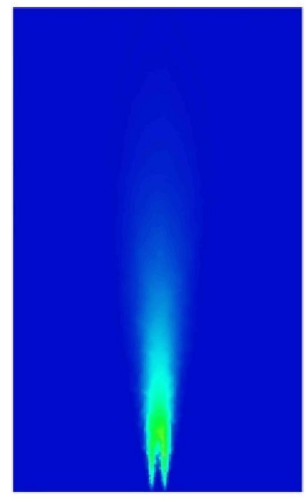

BFLs2 model

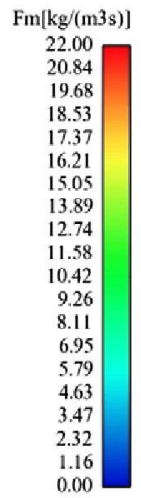

BFL model

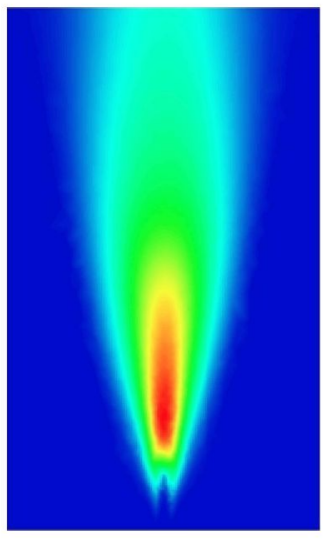

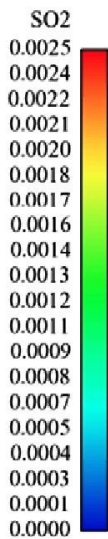

0.0000

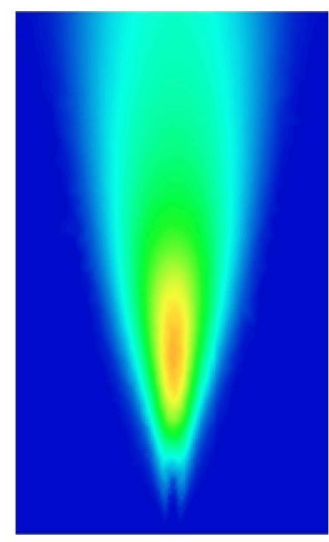

BFLs1 model
Fig. 9. Homogenized mass source $\left(\mathrm{kg} /\left(\mathrm{m}^{3} \mathrm{~s}\right)\right)$.
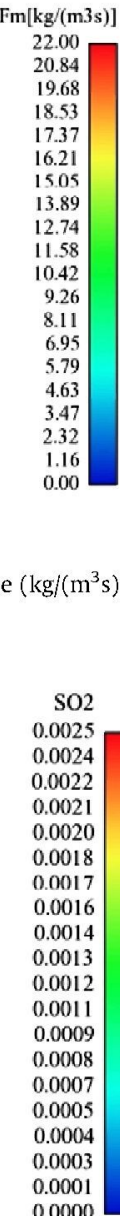

0.0000

Fig. 10. Mass fraction of $\mathrm{SO}_{2}$.

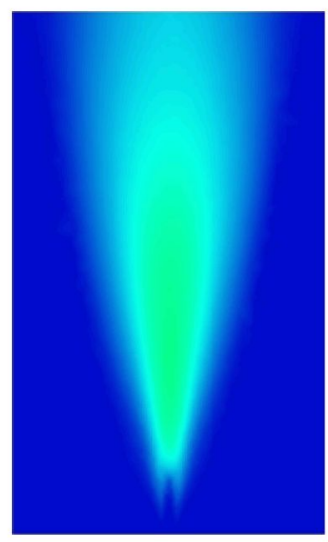

$\mathrm{SO} 2$

0.0025

0.0022

0.0021

0.0020

0.0018

0.0017

0.0016

0.0014

0.0013

0.0012

0.0011

0.0009

0.0008

0.0007

0.0005

0.0004

0.0003

0.0001

0.0000

BFLs2 model

with our three models. The first peak in the axial temperature distribution corresponds to the upstream branch of the main diffusion flame while the second peak corresponds to the downstream branch of the diffusion flame. This does not appear in the BFLs2 model where the second peak is not seen because of the interruption of the computational domain. Similar peaks are found in the radial temperature distribution. These peaks in the mean gas temperature distribution are not smooth in our calculations because we do not used a presumed PDF to model the turbulent fluctuations of the conserved scalars, in particular $\beta_{1}^{g}$. Our calculated peaks in the temperature should be close to the peaks encountered in the instantaneous fluctuating temperature profiles. Notice also the variation along the diffusion flame of the temperature, from the upstream to the downstream branches of the flame.

Let us recall that the main difference between the BFL and the BFLs1 models lies in that for the latter the diffusion flame sur- 


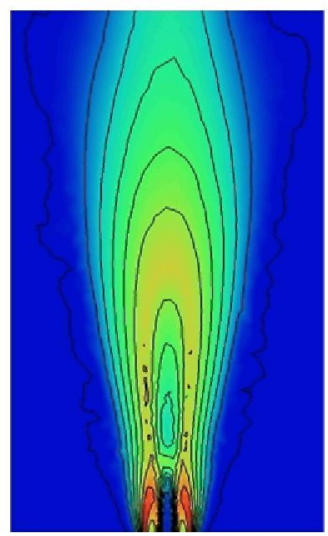

BFL model

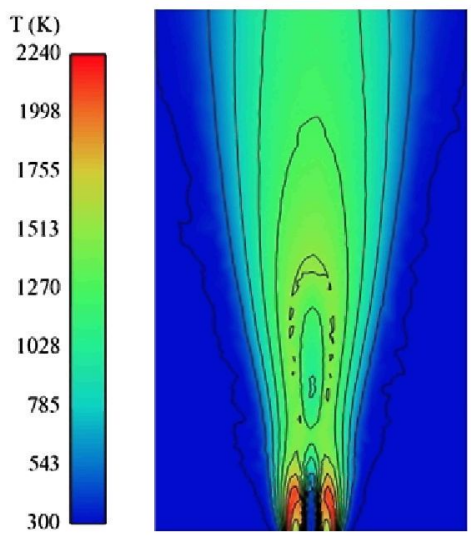

BFLs1 model

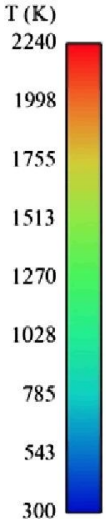

300

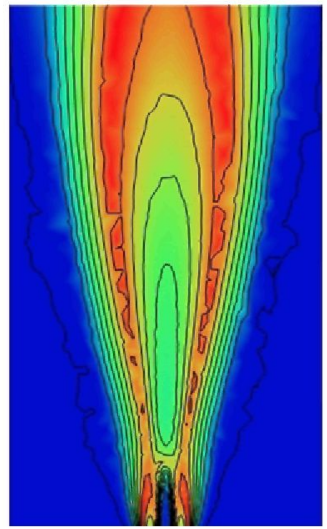

BFLs2 model

Fig. 11. Temperature (K) of the gas mixture.

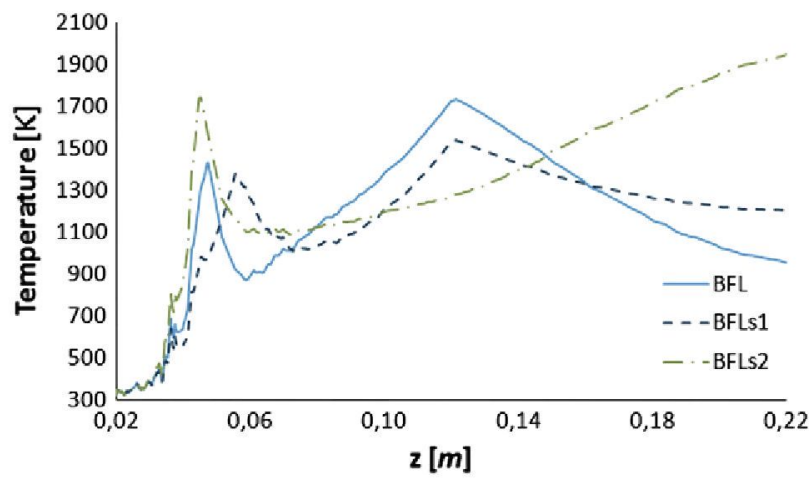

Fig. 12. Numerical axial distribution of gas temperature.

rounding each particle does not occur. However, the numerical results are quite similar. One of the reasons for this behavior is the small value of the diffusion coefficient $\mathcal{D}_{e}$ in the particle. In both models, the inhibitory effect of the ash shell on the rate of arrival to the char by diffusion of the gasifying species slows the rate of generation of $\mathrm{CO}$ and $\mathrm{H}_{2}$, and then of the particle heating associated to their oxidation. Soon, the cooling effect of the ambient air entrainment dominates the variation of the gas temperature and also of the particle temperature. Thus both models lead to high values of the unburnt char, because when the temperature of the particles falls below the critical temperature $T_{C}$ the char gasification is quenched. Even so, some differences can be observed in the two models: due to the presence of the diffusion flame in the interior or in the close vicinity of the particle in the BFL model, the temperature of the particle rises more quickly than in the BFLs1 model. As a consequence, more char is gasified earlier and thus the region $\Omega_{F}$ is closer to the injector.

When we analyze the results provided by the BFLs 2 model great differences with respect to the other two models are observed. The

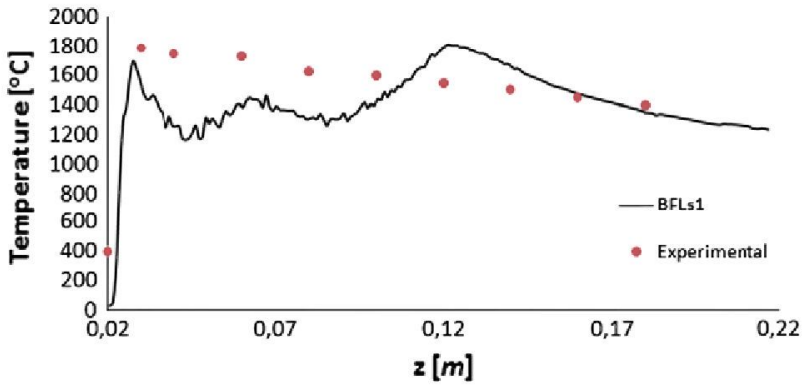

Fig. 14. Distribution of particle temperature on the central axis.

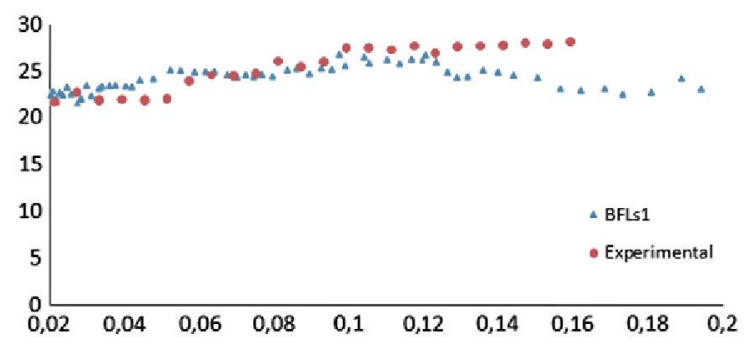

Fig. 15. Distribution of mean diameter on the central axis.

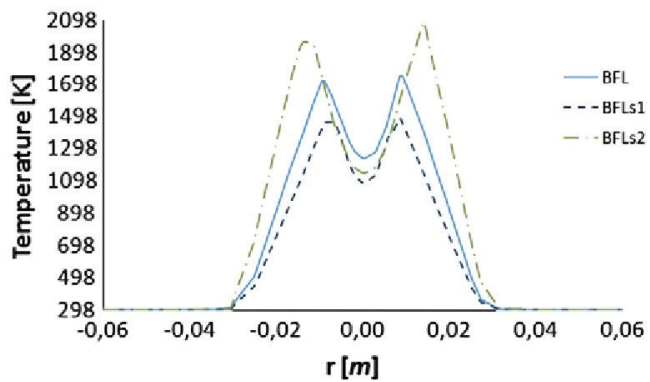

Temperature obtained with our three models

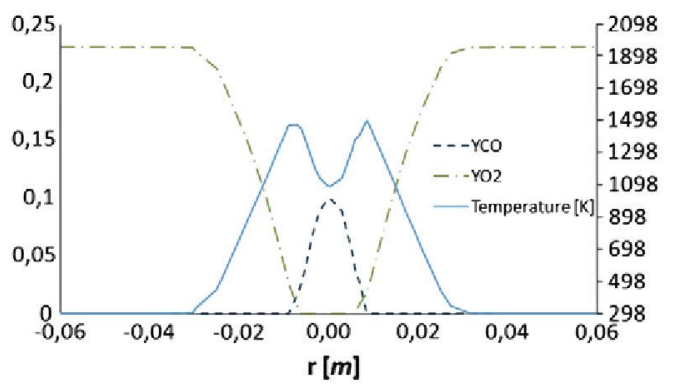

Values obtained with the BFLs1 model

Fig. 13. Numerical radial distribution of gas temperature and mass fractions of $O_{2}$ and $\mathrm{CO}$ at $z=90 \mathrm{~mm}$. 
assumption of disruption of the ash layer leads to a higher rate of char gasification, enough to maintain longer the particle temperature above the critical, thus extending the char gasification until it is almost completely gasified. In view of these results we can conclude that the BFLs2 model is not appropriate to describe the results of this experiment, and a $15 \%$ of ash content cannot be considered as low enough to justify the ash layer disruption.

In the following we will compare the experimental results with the numerical results carried out with the BFLs1 model, which is the best adapted to the experiments. We first compare in Fig. 14 our results for the temperature of the particles with the experimental one appearing in $[12,13,24]$. In these papers the authors show the temperature as measured with a two color radiation pyrometer; however, as the authors say, these measures are imprecise and represent the temperature of gas and particles in a relative large region around the axis.

Figure 15 shows the axial distribution of mean diameters, whereas Fig. 16 shows the radial distributions of the mean diameters at distances $z=60,120$ and $180 \mathrm{~mm}$. These are compared with experimental values appearing in [24]. It can be seen that the particle dispersion is over-predicted in our simulation based on the $k-\epsilon$ model. In fact, one can see that the mean diameter is greater the farther you are from the burner to a distance of $120 \mathrm{~mm}$. This is because smaller particles are more dispersed and therefore away from the axis. However, from this location onwards the mean diameter begins to decrease slightly, unlike what happens with the experimental data. This fact could be explained because the BFLs 1 model does not consider changes in particle sizes, due to partial disruption or swelling effects, and also because turbulent dispersion of the particles is overestimated and hence larger particles are more dispersed. This is also shown in Fig. 16 where we can observe that as we move away from the burner, the diameter distribution tends to be uniform.

Finally, we show in Fig. 17 the particle size distribution at the axis, at distances $z=60,120$ and $180 \mathrm{~mm}$ from the inlet. They are compared with those shown in the first row of Fig. 17 in [24]. As we have advanced before, we could not expect a better agreement between numerical predictions and these experimental data.

\section{Conclusions}

The purpose of this paper is to complete and extend the methodology developed by Bermúdez et al. [11] for the mathematical modeling of combustion of pulverized coal particles, and to show its applicability for the understanding of the results of some of the experiments dealing with a pulverized coal jet flame carried out by Hwang et al. in $[12,13,24]$. In these experiments a circular jet of pulverized coal and ambient temperature air, as carrier gas, exhausts into nearly stagnant air, also at ambient temperature. The coal jet and the ambient air are initially separated by a small annular methane jet. After the methane/air diffusion flame ignites, the combustion products heat the injected gas and, thus, the coal particles. These, when heated, act as distributed sources of volatiles, $\mathrm{CO}$ and $\mathrm{H}_{2}$. In our simulation these species burn with the oxygen of the heated air, as soon as they are supplied to the gas. After the initial region of distributed gas phase oxidation reactions, the oxygen is completely consumed and the gasifying particles enter a region $\Omega_{F}$ without oxygen. This region is enclosed by $\Gamma_{F}$, where the species gasified inside burn in a diffusion-controlled way with the oxygen coming from the outer ambient air.

In the experiments the outer air is at ambient temperature and when entrained into the jet lowers the gas and, later, the particle temperature to quench the char gasification reactions; thus, the particles leave the burner with a large fraction of ungasified char. This large fraction is reproduced only when we take into account, in the models BFL and BFLs1, the effect of an ash shell surrounding the particles that grows during the char gasification.

Two simple variants of the model proposed in [11] have been introduced. Numerical tools for solving the different equations were implemented. The results obtained with the three models were compared. Furthermore, numerical results obtained with the best adapted model were compared with the experimental ones, showing a good agreement. In order to better compare the experimental results and those given by our models more reliable measurements of the temperature of the gas and of the particles would be required. Also, our models, which are appropriate for the direct numerical simulation of pulverized coal combustion with fast gas phase reactions, must be extended to deal with the

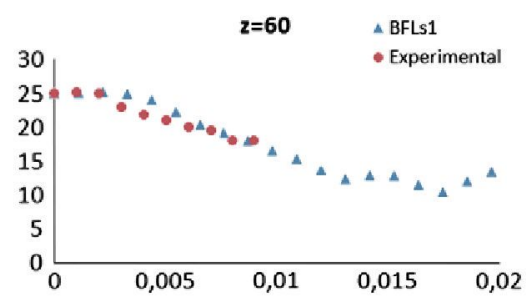

(a) $z=60 \mathrm{~mm}$

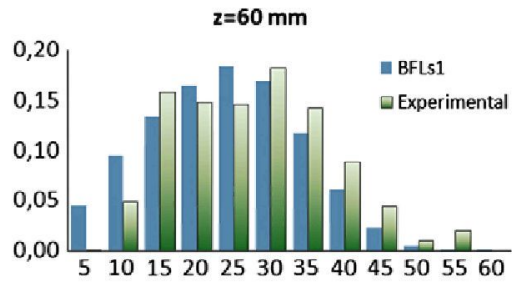

(a) $z=60 \mathrm{~mm}$

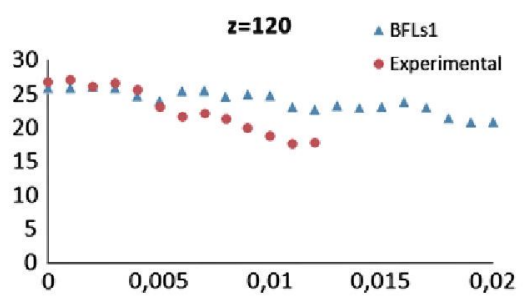

(b) $z=120 \mathrm{~mm}$

Fig. 16. Radial distribution of mean diameters.

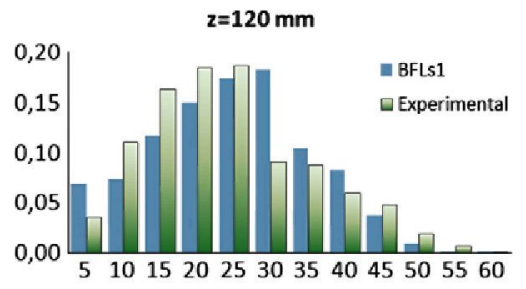

(b) $z=120 \mathrm{~mm}$

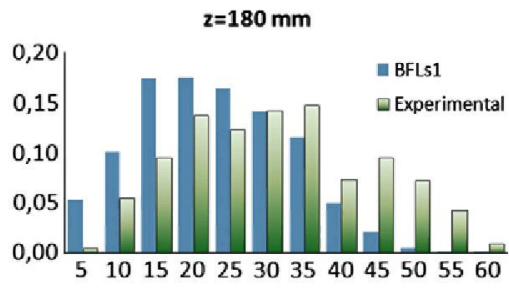

(c) $z=180 \mathrm{~mm}$

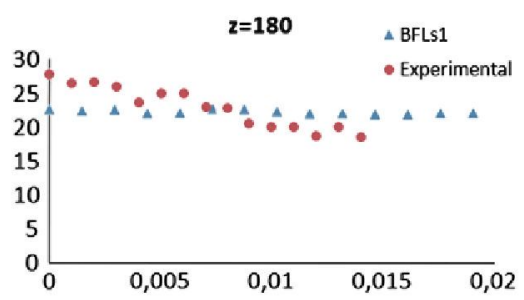

(c) $z=180 \mathrm{~mm}$

Fig. 17. Particle size distributions on the axis. 
evaluation of the mean values of the temperature and concentration distributions in turbulent combustion. This extension would be based on the derivation of appropriate presumed PDF models of the conserved scalars. This turbulent BFL model requires future work.

\section{Acknowledgments}

This work was partially supported by the Spanish MICINN projects ENE2005-09190-C04-01/CON and MTM2008-02483, and the CONSOLIDER project SCORE. It was also supported by the Fundación Ciudad de la Energía under project SIMULOX, by the MEC under the CONSOLIDER project $\mathrm{i}-\mathrm{MATH}$ and by the Comunidad de Madrid under contract HYSYCOMB, S2009/ENE-1597.

\section{References}

[1] Annual Energy Review 2008. DOE/EIA-0384(2008), US Department of Energy, Energy Information Agency, 2009.

[2] F.C. Lockwood, A.P. Salooja, S.A. Syed, Combust. Flame 38 (1980)

[3] R.K. Boyd, J.H. Kent, Proc. Combust. Inst. 21 (1988) 265-274.

[4] S.C. Hill, L.D. Smoot, Energy Fuels 7 (6) (1993) 874-883.

[5] J.S. Anagnostopoulos, N.P. Sargianos, G. Bergeles, Combust. Flame 92 (3) (1993) $209-221$.

[6] U. Schnell, R. Schneider, H.C. Magel, B. Risio, J. Lepper, K.R.G. Hein, Numerica] simulation of advanced coal-fired combustion systems with in-furnace $\mathrm{NO}_{x}$ control technologies, in: Proceedings of the 3 th International Conference on Technologies and Combustion for Clean Environment, Lisbon, Portugal, 1995, pp. 1-11.
[7] A. Bermúdez, J.L. Ferrín, Modelling and numerical solution of a pulverized coal furnace, in: Proceedings of the 4th International Conference on Technologies and Combustion for Clean Environment, Lisbon, Portugal, 1997, pp. 1-9 (paper 33.1).

[8] A.M. Eaton, L.D. Smoot, S.C. Hill, C.N. Eatough, Prog. Energy Combust. Sci 25 (4) (1999) 387-436.

[9] E. Korytnyi, R. Saveliev, M. Perelman, B. Chudnovsky, E. Bar-Ziv, Fuel 88 (1) (2009) 9-18.

[10] A. Williams, R. Backreedy, R. Habib, J.M. Jones, M. Pourkashanian, Fuel 81 (5) (2002) 605-618.

[11] A. Bermúdez, J.L. Ferrîn, A. Liñán, Combust. Theory Model 11 (6) (2007) 949976.

[12] S. Hwang, R. Kurose, F. Akamatsu, H. Tsuji, H. Makino, M. Katsuki, JSME Int. J. Ser. B 49 (4) (2006) 1316-1327.

[13] S. Hwang, R. Kurose, F. Akamatsu, H. Tsuji, H. Makino, M. Katsuki, JSME Int. J. Ser. B 49 (4) (2006) 1328-1335.

[14] S.K. Bhatia, Chem. Eng. Sci. 46 (5/6) (1991) 1465-1474.

[15] G.R. Gavalas, Combust. Sci. Technol. 24 (5) (1980) 197-210.

[16] G.B. Wallis, One Dimensional and Two-phase Flow, McGraw-Hill, New York, 1969.

[17] L. Tian, G. Ahmadi, J. Aerosol. Sci 38 (4) (2007) 377-397.

[18] A.D. Gosman, E. Ioannides, J. Energy 7 (6) (1983) 482-490.

[19] Q. Zhou, M.A. Leschziner, A time-correlated stochastic model for particle dispersion in anisotropic turbulence, in: 8th Turbulent Shear Flows Symposium, Munich, 1991.

[20] J.M. Macinnes, F.V. Bracco, Phys. Fluids 12 (1992) 2809-2824.

[21] E.A. Hennick, M.F. Lightstone, Energy Fuels 14 (2000) 95-103.

[22] A. Allievi, R. Bermejo, J. Comput. Phys. 132 (2) (1997) 157-166.

[23] A. Bermúdez, J.L. Ferrín, A. Liñan, L. Saavedra, Mathematical modelling of coal particles combustion in pulverised coal furnaces, in: Progress in Industrial Mathematics at ECMI 2006, Math. Ind., vol.12, Springer, Berlin, 2008, pp. 277283.

[24] S. Hwang, R. Kurose, F. Akamatsu, H. Tsuji, H. Makino, M. Katsuki, Energy Fuels 19 (2005) $382-392$. 